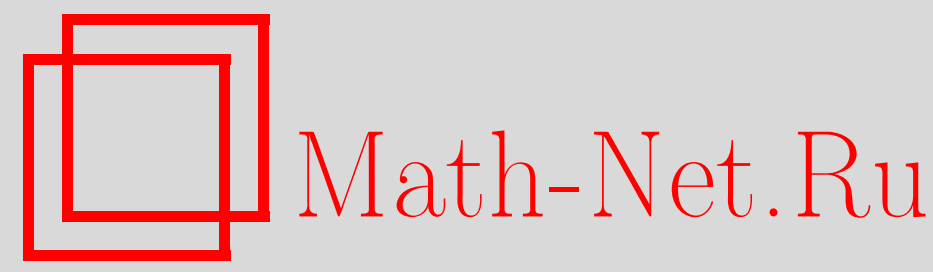

Ю. Б. Орочко, Некоторые свойства индексов дефекта симметрических вырождающихся эллиптических операторов второго порядка в $L^{2}\left(\mathbb{R}^{m}\right)$, Изв. РАН. Сер. матем., 1997, том 61, выпуск 5, 71-98

DOI: https://doi.org/10.4213/im152

Использование Общероссийского математического портала Math-Net.Ru подразумевает, что вы прочитали и согласны с пользовательским соглашением http://www . mathnet.ru/rus/agreement

Параметры загрузки:

IP : 54.80 .73 .141

26 апреля 2023 г., 12:26:07 
УДК 517.9

\author{
Ю. Б. Орочко
}

\title{
Некоторые свойства индексов дефекта симметрических вырождающихся эллиптических операторов второго порядка в $L^{2}\left(\mathbb{R}^{m}\right)$
}

Пусть $H$ - минимальный оператор в $L^{2}\left(\mathbb{R}^{m}\right), \quad m \geqslant 2$, порожденный действительным формально самосопряженным вырождающимся эллиптическим ДВ (дифференциальным выражением) второго порядка $\mathscr{L}$. На примере оператора $H=H_{0}$, отвечающего ДВ $\mathscr{L}=\mathscr{L}_{0}=-\operatorname{div} a(|x|) \operatorname{grad}$, где $a(r)$, $r \in[0,+\infty),-$ скалярная неотрицательная функция, устанавливается зависимость значений индексов дефекта $H$ от степени гладкости старших коэффициентов $\mathscr{L}$. Вторым результатом, полученным в работе, является признак самосопряженности оператора $H$, в котором отсутствуют какие-либо ограничения на поведение потенциала ДВ $\mathscr{L}$ при $|x| \rightarrow+\infty$. Эти результаты не имеют прямых аналогов в случае эллиптичности ДВ $\mathscr{L}$.

Библиограффия: 18 наименований.

\section{Введение}

Характерной чертой известных признаков самосопряженности минимального оператора $H$ в пространстве $L^{2}\left(\mathbb{R}^{m}\right), m \geqslant 2$, порожденного эллиптическим ДВ (дифференциальным выражением)

$$
\mathscr{L}=-\operatorname{div} A(x) \operatorname{grad}+q(x)
$$

с действительной симметрической положительно определенной (в каждой точке $x \in \mathbb{R}^{m}$ ) матрищей $A(x)=\left\{a_{j k}(x)\right\}_{j, k=1}^{m}$ и действительным потенциалом $q(x)$ является обязательное присутствие в них тех или иных ограничений на $A(x)$ и $q(x)$ в окрестности бесконечно удаленной точки. В исследованиях 60-70-х годов выяснилось, что имевшиеся в первоначальных вариантах этих результатов (и представлявшиеся естественными) требования принадлежности старших коэффициентов $a_{j k}(x)$ классу $C^{1}\left(\mathbb{R}^{m}\right)$ можно ослабить; вместо указанного локального ограничения достаточно потребовать, например, выполнения условий

$$
a_{j k}(x) \in C\left(\mathbb{R}^{m}\right), \quad \frac{\partial a_{j k}}{\partial x_{j}}(x) \in\left(L_{p} \cap L_{2}\right)_{\operatorname{loc}}\left(\mathbb{R}^{m}\right), \quad p>m
$$

Работа выполнена при частичной финансовой поддержке Международного научного фонда (грант № 3І000) и РФФИ (грант № 96-01-01438).

(C) Ю.Б. Орочко 1997 
В настоящей работе рассматриваются вопросы, относящиеся к условиям самосопряженности оператора $H$ в случае вырождаюшейся эллиптичности ДВ $\mathscr{L}$. Мы хотим обратить внимание на два новых явления, возникающие в этой ситуации. Одно из них состоит в зависимости значений индексов дефекта оператора $H$ от степени гладкости старших коэффищиентов $a_{j k}(x) \in C\left(\mathbb{R}^{m}\right)$ вырождаюшегося эллиптического ДВ $\mathscr{L}$, а другое - в существовании условий самосопряженности минимального оператора, отвечающего такому ДВ, в котором нет никаких ограничений на поведение его потенциала при $|x| \rightarrow+\infty$.

Результаты, относящиеся к первому из указанных явлений (теоремы 1-3), изложены в $\S 1$. Второму из них посвяшен $\S 2$, основным результатом которого является теорема 4. Теорема 4 имеет связь с интересным явлением неустойчивости свойства несамосопряженности оператора $H$ (в эллиптическом и вырождающемся эллиптическом случае) относительно вариации старших коэффициентов ДВ $\mathscr{L}$ на множествах сколь угодно малой положительной меры в $\mathbb{R}^{m}$. Эта связь кратко обсуждается в п. 2.5.

\section{§1. Пример зависимости индексов дефекта минимального вырождающегося эллиптического оператора от степени гладкости его старших коэффициентов}

1.1. Рассмотрим простейшее ДВ

$$
\mathscr{L}_{0}=-\operatorname{div} a(|x|) \operatorname{grad}, \quad x \in \mathbb{R}^{m}, \quad m \geqslant 2,
$$

скалярный коэффициент которого $a(|x|)$ связан соотношением

$$
a(r)=|b(r)|
$$

с функцией $b(r)$, удовлетворяющей условиям:

I) $b(r) \in C^{2}([0,+\infty))$;

II) существует число $r_{0}>0$ такое, что

$$
\begin{gathered}
b(r)>0, \quad r \in\left[0, r_{0}\right), \\
|b(r)|>0, \quad r \in\left(r_{0},+\infty\right), \\
b\left(r_{0}\right)=0 .
\end{gathered}
$$

Таким образом, $r_{0}$ является единственным нулем функции $b(r)$ на полуоси $[0,+\infty)$. Из формулы $(3)$ следует, что ДВ $\mathscr{L}_{0}$ обладает свойством вырождающейся эллиптичности в $\mathbb{R}^{m}$, причем множеством его точек вырождения является сфера $S_{r_{0}}$ (символом $S_{\rho}$ в настояшей работе обозначается сфера $|x|=\rho$ в $\mathbb{R}^{m}$ радиуса $\rho>0)$.

Если функция $b(r)$ удовлетворяет условиям I), II), то либо

$$
b\left(r_{0}\right)=0, \quad b^{\prime}\left(r_{0}\right)=0, \quad b^{\prime \prime}\left(r_{0}\right)>0,
$$


либо

$$
b\left(r_{0}\right)=0, \quad b^{\prime}\left(r_{0}\right)<0 .
$$

В первом случае $a(r)=b(r), r \in[0,+\infty)$, и поэтому $a(r) \in C^{2}([0,+\infty))$. Если же выполнены условия (9), то $b(r)<0$ при $r \in\left(r_{0},+\infty\right), a(r)=$ $\left|b^{\prime}\left(r_{0}\right)+o(1)\right|\left|r-r_{0}\right|$ в некоторой окрестности точки $r_{0}$. Следствием последней формулы является недифференцируемость функции $a(r)$ в точке $r_{0}$; вместе с тем $a(r) \in C^{2}\left(\left[0, r_{0}\right) \cup\left(r_{0},+\infty\right)\right)$. Сделанные замечания приводят к выводу, что коэффициент $a(|x|)$ ДВ $\mathscr{L}_{0}$, связанный с функцией $b(r)$, удовлетворяющей условиям I), II), равенством (3), принадлежит классу $C^{2}\left(\mathbb{R}^{m} \backslash\{0\}\right) \cap C\left(\mathbb{R}^{m}\right)$, если выполнены соотношения (8) (при дополнительном условии $b^{\prime}(0)=0$ можно утверждать, что $\left.a(|x|) \in C^{2}\left(\mathbb{R}^{m}\right)\right)$. Если выполняются соотношения (9), то функция $a(|x|)$ недифференцируема в точках сферы $S_{r_{0}}$ и принадлежит классу $C^{2}\left(\mathbb{R}^{m} \backslash S_{r_{0}}\right) \cap C\left(\mathbb{R}^{m}\right)$ либо классу $C^{2}\left(\mathbb{R}^{m} \backslash\left(S_{r_{0}} \cup\{0\}\right)\right) \cap C\left(\mathbb{R}^{m}\right)$ в зависимости от того, выполняется или не выполняется равенство $b^{\prime}(0)=0$.

На протяжении всей статьи мы понимаем минимальньй оператор в $L^{2}\left(\mathbb{R}^{m}\right)($ а также в пространствах $\left.L^{2}(\Omega), \Omega \subset \mathbb{R}^{m}\right)$, порожденный каким-нибудь $m$-мерным ДВ, как замыкание оператора с областью определения $C_{0}^{\infty}\left(\mathbb{R}^{m}\right)$, действие которого на функции этого класса совпадает с действием на них рассматриваемого ДВ.

Пусть $H_{0}$ - действующий в $L^{2}\left(\mathbb{R}^{m}\right)$ симметрический и неотрищательный минимальньй оператор, порожденный ДВ $\mathscr{L}_{0}$. Его свойства, интересуюшие нас, состоят в следующем.

ТЕОРема 1. Если для функиии b(r), удовлетворяющей условиям I), II), в точке $r_{0}$ дополнительно выполнены условия (8), то оператор $H_{0}$ самосопряжен (т.е. его индексы дефекта равны нулю).

Teорема 2. Оператор $H_{0}$ имеет бесконечные индексы дефекта, если наряду с условиями I), II) функция $b(r)$ в точке $r_{0}$ удовлетворяет условию (9).

Доказательству теоремы 1 посвящены пп. 1.2-1.4; доказательство теоремы 2 излагается в пп. $1.5,1.6$; п. 1.7 посвяшен некоторым замечаниям, относящимся к теоремам 1,2 .

1.2. Доказательство теоремы 1 состоит из двух частей. В первой из них приводится ее подробное обоснование при следующих дополнительных ограничениях:

$(\alpha) b(r) \in C^{\infty}([0,+\infty)), \quad b^{(2 j+1)}(0)=0, \quad j=0,1,2, \ldots ;$

( $\beta) \lim _{r \rightarrow+\infty} b(r)=0$;

$(\gamma)$ функция $a(r)=|b(r)|$ монотонно убывает на полуоси $\left(r_{1},+\infty\right)$ при некотором $r_{1}>r_{0}$.

Во второй части $(п$ п. 1.3, 1.4) мы более сжато опишем технические средства, с помощью которых можно избавиться от указанных дополнительных ограничений, т.е. получить теорему 1 в полной обшности.

Нам понадобятся вспомогательные понятия и обозначения. Будем обозначать через $B_{\rho}$ открытый шар $|x|<\rho$ в $\mathbb{R}^{m}$. Для любой области $\Omega \subset \mathbb{R}^{m}$ мы будем 
рассматривать пространство $L^{2}(\Omega)$ как подпространство в $L^{2}\left(\mathbb{R}^{m}\right)$, состояшее из функций $f(x) \in L^{2}\left(\mathbb{R}^{m}\right)$, равных нулю в $\mathbb{R}^{m} \backslash \Omega$. Отметим очевидное равенство

$$
L^{2}\left(\mathbb{R}^{m}\right)=L^{2}\left(B_{\rho}\right) \oplus L^{2}\left(\mathbb{R}^{m} \backslash \bar{B}_{\rho}\right)
$$

$\left(\oplus\right.$ - знак ортогональной суммы подпространств в $\left.L^{2}\left(\mathbb{R}^{m}\right)\right)$. Класс $C_{0}^{\infty}(\Omega)$ мы также будем рассматривать как подмножество класса $C_{0}^{\infty}\left(\mathbb{R}^{m}\right)$ (в указанномвьше смысле).

Пусть $P, Q$ - минимальные симметрические неотрицательные операторы в $L^{2}\left(B_{r_{0}}\right)$ и $L^{2}\left(\mathbb{R}^{m} \backslash \bar{B}_{r_{0}}\right)$, порожденные ДВ $\mathscr{L}_{0}$. Их ортогональная сумма $P \oplus Q$ является замыканием своего сужения на $C_{0}^{\infty}\left(B_{r_{0}}\right) \oplus C_{0}^{\infty}\left(\mathbb{R}^{m} \backslash \bar{B}_{r_{0}}\right) \subset C_{0}^{\infty}\left(\mathbb{R}^{m}\right)$, вследствие чего оператор $H_{0}$ можно рассматривать как симметрическое расширение симметрического оператора $P \oplus Q$. В силу этого замечания для доказательства теоремы 1 достаточно показать, что из ее условий вытекает самосопряженность каждого из операторов $P, Q$. Установим справедливость последнего свойства при дополнительных ограничениях.

ЛЕмма 1. Если наряду с условиями I), II) и (8) функция $b(r)$ удовлетворяет дополнительным ограничениям $(\alpha),(\beta),(\gamma)$, то каждый из неотрицательных симметрических операторов $P, Q$ самосопряжен (в соответствующем подпространстве пространства $L^{2}\left(\mathbb{R}^{m}\right)$ ).

Для доказательства самосопряженности оператора $Q$ возьмем произвольную функцию $f_{0}(x) \in C_{0}^{\infty}\left(\mathbb{R}^{m} \backslash \bar{B}_{r_{0}}\right)$. Для нее существуют числа $k, l, r_{0}<k<l$, такие, что supp $f_{0} \subset B_{l} \backslash B_{k}$. Следствием условия (8) является оценка

$$
0<a(r) \leqslant C\left(r-r_{0}\right)^{2}, \quad 0<\left|r-r_{0}\right|<\varepsilon
$$

( $\varepsilon$ и $C$ - некоторые положительные константы), влекущая за собой свойство

$$
\int_{r_{0}}^{k} a^{-1 / 2}(r) d r=+\infty
$$

Оно означает, что для любой (сколь угодно большой) константы $N>0$ сушествует число $\rho_{N} \in\left(0, k-r_{0}\right)$, для которого

$$
J\left(\rho_{N}\right)=\int_{k-\rho_{N}}^{k} a^{-1 / 2}(r) d r>N .
$$

Благодаря дополнительным ограничениям $(\beta)$ и $(\gamma)$ можно найти число $l_{N}>\max \left\{l, r_{1}\right\}$ такое, что

$$
\max _{\left[l_{N}, l_{N}+\rho_{N}\right]} a(r)<\min _{\left[k-\rho_{N}, k\right]} a(r) .
$$

Рассмотрим уравнение

$$
u_{t t}+\mathscr{L}_{0}[u]=0,
$$


имеющее гиперболический характер в области $\left(\mathbb{R}^{m} \backslash \bar{B}_{r_{0}}\right) \times[0,+\infty) \subset \mathbb{R}^{m+1}$; заметим, что коэффициенты этого уравнения бесконечно дифференцируемы в ней в силу дополнительного ограничения (10). Будем обозначать символом $\operatorname{supp}_{x} u(x, t)$ носитель функции $u(x, t)$ по переменной $x$ при фиксированном $t$. Решение $u(x, t)$ уравнения (16) с начальными условиями

$$
u(x, 0)=f(x) \in C_{0}^{\infty}\left(\mathbb{R}^{m} \backslash \bar{B}_{r_{0}}\right), \quad u_{t}(x, 0)=0
$$

обладает свойством конечной скорости распространения. Поэтому для области $B_{l_{N}} \backslash \bar{B}_{k}$ сушествует наибольшее число $t^{*} \in(0,+\infty]$ такое, что

$$
\operatorname{supp}_{x} u(x, t) \subset \mathbb{R}^{m} \backslash \bar{B}_{r_{0}}, \quad t \in[0,+\infty),
$$

если только начальная функция $f(x)$ удовлетворяет условию

$$
\operatorname{supp} f \subset B_{l_{N}} \backslash \bar{B}_{k} \text {. }
$$

Так как $B_{l} \backslash B_{k} \subset B_{l_{N}} \backslash B_{k}$, то оно заведомо вьполнено для взятой нами функции $f_{0}(x)$. Следовательно, решение $u(x, t)$ задачи Коши $(16),(17)$ с $f(x)=f_{0}(x)$ обладает свойством (18).

Оценку снизу числа $t^{*}$ можно получить, опираясь на лемму 6 работы [1], которая позволяет по множеству $G=B_{l_{N}} \backslash \bar{B}_{k}$ и его окрестности $B_{l_{N}+\rho_{N}} \backslash B_{k-\rho_{N}}$ радиуса $\rho_{N}$ явно указать число $t_{0}$, обеспечивающее свойство

$$
\operatorname{supp}_{x} u(x, t) \subset B_{l_{N}+\rho_{N}} \backslash \bar{B}_{k-\rho_{N}} \subset \mathbb{R}^{m} \backslash \bar{B}_{r_{0}}, \quad t \in\left[0, t_{0}\right),
$$

решения задачи Коши (16), (17) с дополнительным условием (19). С учетом того, что ДВ $\mathscr{L}_{0}$ можно представить в виде (1) с матрицей старших коэффициентов $A(x)=a(|x|) E(E-$ единичная $(m \times m)$-матрица $)$ и потенциалом $q(x) \equiv 0$, а также с учетом равенства (15) формула для $t_{0}$, полученная в [1], в рассматриваемом случае дает значение $t_{0}=J\left(\rho_{N}\right)>N$. Так как очевидным является неравенство $t_{0} \leqslant t^{*}$, то мы приходим к оценке $t^{*} \geqslant N$ для любого сколь угодно большого числа $N>0$, из которого следует, что $t^{*}=+\infty$. Основываясь на этом результате, получаем, что решение $u(x, t)$ задачи Коши $(16),(17)$ с начальной функцией $f(x)=f_{0}(x)$ обладает свойством (18) со значением $t^{*}=+\infty$. По предположению $f_{0}(x)$ - произвольная функция класса $C_{0}^{\infty}\left(\mathbb{R}^{m} \backslash \bar{B}_{r_{0}}\right)$. Следовательно, решение $u(x, t)$ задачи Коши $(16),(17)$ обладает свойством (18), какова бы ни была начальная функция $f(x) \in C_{0}^{\infty}\left(\mathbb{R}^{m} \backslash \bar{B}_{r_{0}}\right)$. При этом из бесконечной дифференцируемости коэффищиентов уравнения $(16)$ в $\left(\mathbb{R}^{m} \backslash \bar{B}_{r_{0}}\right) \times[0,+\infty)$ вытекает, что $u(x, t) \in C^{\infty}\left(\left(\mathbb{R}^{m} \backslash B_{r_{0}}\right) \times[0,+\infty)\right)$.

Перечисленные свойства решения $u(x, t)$ задачи Коши $(16),(17)$ позволяют утверждать, что если $u(x, t)$ рассматривать как вектор-функцию $u(t)=u(\cdot, t)$ параметра $t$ со значениями в $L^{2}\left(\mathbb{R}^{m} \backslash \bar{B}_{r_{0}}\right)$, то такая вектор-функция обладает свойствами

$$
\begin{gathered}
u(t) \in C_{0}^{\infty}\left(\mathbb{R}^{m} \backslash \bar{B}_{r_{0}}\right) \subset \mathscr{D}(Q), \quad t \in[0,+\infty) ; \\
u(0)=f \in C_{0}^{\infty}\left(\mathbb{R}^{m} \backslash \bar{B}_{r_{0}}\right), \quad u^{\prime}(0)=0 ; \\
u^{\prime \prime}(t)+Q u(t)=0, \quad t \in[0,+\infty)
\end{gathered}
$$


(на протяжении всей статьи производные вектор-функции параметра $t$ co значениями в гильбертовом пространстве понимаются в сильном смысле). Так как симметрический оператор $Q$ неотрищателен, то у него есть фридрихсово самосопряженное расширение

$$
F \supseteq Q, \quad \inf F \geqslant 0 \text {. }
$$

Так как свойство (22) можно переписать в виде $u^{\prime \prime}(t)+F u(t)=0, t \in[0,+\infty)$, то с учетом начальных условий (21) оно приводит к представлению

$$
u(t)=\cos \left(t F^{1 / 2}\right) f, \quad t \in[0,+\infty),
$$

следствием которого является оценка нормы

$$
\|u(t)\| \leqslant\|f\|, \quad t \in[0,+\infty) .
$$

Согласно теореме 1 работы [2] существование для каждой функции $f(x) \in$ $C_{0}^{\infty}\left(\mathbb{R}^{m} \backslash \bar{B}_{r_{0}}\right)$ решения задачи Коши (21), (22), удовлетворяющего такой оценке, обеспечивает самосопряженность оператора $Q$.

Доказательство самосопряженности оператора $P$, действуюшего в $L^{2}\left(B_{r_{0}}\right)$, проводится с помощью рассуждений, аналогичных вышеприведенным. Их центральным местом является обоснование свойства

$$
\operatorname{supp}_{x} \tilde{u}(x, t) \subset B_{r_{0}}, \quad t \in[0,+\infty),
$$

решения $\tilde{u}(x, t)$ уравнения (16), удовлетворяющего начальным условиям $\tilde{u}(x, 0)=$ $\tilde{f}(x) \in C_{0}^{\infty}\left(B_{r_{0}}\right), \quad \tilde{u}_{t}(x, 0)=0$. Если $\tilde{f}(x) \in C_{0}^{\infty}\left(B_{r_{0}}\right)$, то $\operatorname{supp} f \subset B_{\tau}$ для некоторого $\tau \in\left(0, r_{0}\right)$. Оценка $(12)$ влечет за собой расходимость интеграла

$$
\int_{\tau}^{r_{0}} a^{-1 / 2}(r) d r
$$

(аналог формулы (13)); поэтому для произвольной константы $N>0$ можно подобрать такое число $\rho_{N} \in\left(0, r_{0}-\tau\right)$, что выполняется неравенство

$$
\int_{\tau}^{\tau+\rho_{N}} a^{-1 / 2}(r) d r>N,
$$

аналогичное неравенству (14). Свойство (26) является следствием леммы 6 работы [1], если ее применить к множеству $G=B_{\tau}$ и его окрестности $B_{\tau+\rho_{N}} \subset B_{r_{0}}$ радиуса $\rho_{N}$. С учетом свойства $(26)$ вектор-функция $\tilde{u}(t)=\tilde{u}(\cdot, t)$ со значениями в $L^{2}\left(B_{r_{0}}\right)$ удовлетворяет условиям:

$$
\begin{gathered}
\tilde{u}(t) \in \mathscr{D}(P) \quad \text { при } \quad t \in[0,+\infty), \\
\tilde{u}(0)=\tilde{f}, \quad \tilde{u}^{\prime}(0)=0, \quad \tilde{u}^{\prime \prime}(t)+P \tilde{u}(t)=0 \quad \text { при } \quad t \in[0,+\infty) .
\end{gathered}
$$

Из них самосопряженность оператора $P$ вытекает точно так же, как из формул (20)-(22) следует самосопряженность оператора $Q$. Лемма 1 доказана; вместе с ней установлена справедливость теоремы 1 при дополнительных ограничениях $(\alpha)$, $(\beta),(\gamma)$. 
1.3. Укажем способ, позволяющий получить теорему 1 в полной общности. Присутствуюшее в лемме 1 ограничение (10), более жесткое, чем требование (4) теоремы 1 , обеспечивает бесконечную дифференцируемость коэффициентов уравнения (16), вследствие чего не возникает сомнений в существовании его классических решений, используемых в доказательстве этой леммы. К сожалению, в случае, когда вместо (10) выполнено условие (4), существование упомянутых классических решений уравнения (16) вытекает из известных результатов теории гиперболических уравнений только при малых размерностях пространства $\mathbb{R}^{m}$ (см $[3$, гл. $3, \S 21])$. Это обстоятельство требует обрашения к обобщенным решениям уравнения (16), если мы хотим получить утверждение леммы 1 при условии (4) вместо (10). В работе [4] для гиперболического уравнения $u_{t t}+\mathscr{L}[u]=0$, в котором $\mathscr{L}$ - эллиптическое ДВ (1) со старшими коэффициентами

$$
a_{j k}(x) \in L_{\mathrm{loc}}^{\infty}\left(\mathbb{R}^{m}\right),
$$

введен класс обобщенных решений, обладающих свойством конечной скорости распространения, вполне аналогичньм свойству конечной скорости распространения классических решений уравнения (16) - основному инструменту доказательства леммы 1 . В частности, для них справедлив аналог вспомогательного результата - леммы 6 работы [1], - с помощью которого были установлены свойства (18) с $t^{*}=+\infty$ и (26) классических решений задачи Коши для уравнения (16) (см. [4, лемма 2.6]).

Так как при выполнении условия (4) коэффициенты ДВ $\mathscr{L}_{0}$, очевидно, обладают свойством (27), то для получения утверждения леммы 1 при ограничении (4), менее жестком, чем (10) (без изменения прочих ее условий), следует повторить рассуждения, приведенные в п. 1.2, заменяя в них классические решения уравнения (16) обобщенными, рассматриваемыми в статье [4]. Единственная существенная поправка, требуемая в этом случае, касается классов $C_{0}^{\infty}\left(\mathbb{R}^{m} \backslash \bar{B}_{r_{0}}\right)$ и $C_{0}^{\infty}\left(B_{r_{0}}\right)$, из которых в п. 1.2 брались начальные значения присутствуюших в формулах (18) с $t^{*}=+\infty$ и (26) классических решений $u(x, t), \tilde{u}(x, t)$ уравнения $(16)$. В качестве начальных значений обобщенных решений этого уравнения - аналогов $u(x, t)$, $\tilde{u}(x, t)$ - следует брать функции с носителем в $\mathbb{R}^{m} \backslash \bar{B}_{r_{0}}$ и соответственно в $B_{r_{0}}$, принадлежашие введенному в статье [4] классу финитных функций Д, конструируемому по ДВ $\mathscr{L}_{0}$. С учетом свойств функционального класса Д, изученных в [4], использование таких начальных функций не вносит существенных осложнений в схему рассуждений п. 1.2.

1.4. Мы объяснили, каким образом оба утверждения леммы 1 (о самосопряженности каждого из операторов $P, Q$ ) могут быть доказаны в случае, когда из присутствующих в ней дополнительных ограничений $(\alpha),(\beta),(\gamma)$ вьполняются только два последние, а ограничение ( $\alpha$ ) заменено условием (4). Заметим, что дополнительные ограничения $(\beta)$ и $(\gamma)$ леммы 1 , относяшиеся к свойствам функции $b(r)$ при $r \rightarrow+\infty$, не использовались в доказательстве самосопряженности оператора $P$, и вместе с тем их следствие - неравенство (15) - играло важную роль в проверке самосопряженности оператора $Q$. Поэтому для доказательства теоремы 1 в 
полной общности достаточно установить справедливость следующего уточнения утверждения леммы 1 о самосопряженности оператора $Q$.

ЛЕмма 2. Пусть функция $b(r)$ удовлетворяет только условиям I), II) (заметим, что в них нет ограничений на поведение функиии $b(r) \in C^{2}([0,+\infty))$ при $r \rightarrow+\infty$, кроме требования ее знакоопределенности на полуоси $\left.\left(r_{0},+\infty\right)\right)$. Тогда минимальный оператор $Q$ в $L^{2}\left(\mathbb{R}^{m} \backslash B_{r_{0}}\right)$, порожсденный ДВ $\mathscr{L}_{0}$, самосопряжен.

ЗАмЕчаниЕ 1. Доказательство леммы 1 является промежуточным этапом в доказательстве леммы 2. Способ проверки справедливости леммы 2, не использующий леммы 1 , автору неизвестен.

При проверке справедливости леммы 2 мы опираемся на то, что в сферических координатах $r$ (радиальная переменная) и $\theta=\left(\theta_{1}, \theta_{2}, \ldots, \theta_{m-1}\right)$ (угловой параметр) ДВ $\mathscr{L}_{0}$ имеет вид

$$
-r^{-(m-1)} \frac{d}{d r} r^{m-1} a(r) \frac{d}{d r}-\frac{a(r)}{r^{2}} \Delta_{\theta}
$$

где $\Delta_{\theta}$ - выражение оператора Бельтрами-Лапласа на единичной сфере $S_{1}$ через угловые переменные $\theta_{1}, \theta_{2}, \ldots, \theta_{m-1}$. Оператор Бельтрами-Лапласа на $S_{1}$ с областью определения $C^{\infty}\left(S_{1}\right)$ симметричен в пространстве $L^{2}\left(S_{1}, d \sigma\right)$ (через $d \sigma$ обозначен элемент площади поверхности) и имеет чисто дискретный спектр, состоящий из чисел $\varkappa_{l}=-l(l+m-2), l=0,1,2, \ldots$, причем собственное подпространство этого оператора, отвечающее его собственному значению $\varkappa_{l}$, состоит из функций $P_{l}\left(\frac{x}{|x|}\right)$ - значений на $S_{1}$ гармонических многочленов $P_{l}(x)$ порядка $l$ (см., например, $[5$, гл. $3, \S 22])$. Если $h_{l}(\theta)$ - выражение функции $P_{l}\left(\frac{x}{|x|}\right)$ через угловой параметр $\theta$, то последнее свойство означает, что

$$
-\Delta_{\theta} h_{l}=l(l+m-2) h_{l}
$$

Используя разделение переменных в сфрерических координатах (см. [6, гл.10, дополнение к $\S 10.1])$, нетрудно проверить, что оператор $Q$ самосопряжен тогда и только тогда, когда при каждом $l \geqslant 0$ самосопряжен действующий в пространстве $L^{2}\left(\left(r_{0},+\infty\right), r^{m-1} d r\right)$ с весом $r^{m-1}$ минимальный оператор $T_{l}$, порожденный обыкновенным ДВ

$$
\tau_{l}=-r^{-(m-1)} \frac{d}{d r} r^{m-1} a(r) \frac{d}{d r}+l(l+m-2) \frac{a(r)}{r^{2}}
$$

(напомним, что $a(r)= \pm b(r)$ при $r>r_{0}$ ). Так как в лемме 1 самосопряженность оператора $Q$ установлена при выполнении наряду с условиями I), II) теоремы 1 дополнительных ограничений $(\beta)$ и $(\gamma)$ (мы уже объяснили, что от ограничения $(\alpha)$ можно избавиться), то при этих условиях из указанной леммы вытекает самосопряженность оператора $T_{l}$ при любом $l$. Последнее свойство означает, что в указанном случае для индекса дефекта $d_{l}$ (для определенности в верхней комплексной полуплоскости) оператора $T_{l}$ справедливо равенство $d_{l}=0, l \geqslant 0$. 
Зафиксируем какую-нибудь точку $\rho \in\left(r_{0},+\infty\right)$ и обозначим через $d_{l}^{(1)}$ и $d_{l}^{(2)}$ индексы дефекта в верхней комплексной полуплоскости минимальных операторов $T_{l}^{(1)}, T_{l}^{(2)}, \quad$ порожденных ДВ $\tau_{l}$ соответственно в $L^{2}\left(\left(r_{0}, \rho\right), r^{m-1} d r\right)$ и $L^{2}\left((\rho,+\infty), r^{m-1} d r\right)$. Тогда числа $d_{l}, d_{l}^{(1)}, d_{l}^{(2)}$ связаны известной формулой

$$
d_{l}=d_{l}^{(1)}+d_{l}^{(2)}-2
$$

(см. [7, гл. $13, \S 2$, следствие 26$])$; заметим, что пространства $L^{2}\left(J, r^{m-1} d r\right), J \subseteq \mathbb{R}$, переводятся в пространства $L^{2}(J)=L^{2}(J, d r)$, рассматриваемые в [7], простым унитарным преобразованием

$$
L^{2}\left(J, r^{m-1} d r\right) \ni f(r) \rightarrow r^{(m-1) / 2} f(r) \in L^{2}(J)
$$

Один из результатов работы [8] обеспечивает справедливость равенства $d_{l}^{(2)}=1, l \geqslant 0$, при произвольном поведении при $r \rightarrow+\infty$ положительной на $(\rho,+\infty)$ функции $a(r)=|b(r)|$ и, в частности, в случае, когда $b(r)$ удовлетворяет условиям $(\beta)$ и $(\gamma)$. Так как $d_{l}=0, d_{l}^{(2)}=1$, то следствием формулы (31) является равенство $d_{l}^{(1)}=1, l \geqslant 0$. Последнее равенство получено в предположении, что выполнены дополнительные ограничения $(\beta),(\gamma)$ леммы 1 , относящиеся к поведению функции $b(r)= \pm a(r)$ на бесконечности, но ввиду того, что индекс дефекта $d_{l}^{(1)}$ зависит только от свойств функции $a(r)$ на ограниченном интервале $\left(r_{0}, \rho\right)$, формула $d_{l}^{(1)}=1$ верна независимо от поведения $a(r)$ при $r \rightarrow+\infty$. Если функция $b(r)$ удовлетворяет только условиям I), II) и, следовательно, произвольна на бесконечности, то из формул $d_{l}^{(1)}=1, d_{l}^{(2)}=1$ и (31) вытекает, что индексы дефекта соответствуюших операторов $T_{l}$ равны нулю при любом $l \geqslant 0$, и поэтому все эти операторы самосопряжены. Как отмечено выше, это свойство равносильно самосопряженности соответствуюшего оператора $Q$. Лемма 2 , а вместе с ней и теорема 1 доказаны.

ЗАмЕчАнИЕ 2. В доказательстве теоремы 1 главную роль играли два факта, одним из которых является соотношение $H_{0} \supseteq P \oplus Q$, а другим - самосопряженность операторов $P$ и $Q$, и поэтому такое же свойство их ортогональной суммы $P \oplus Q$. Так как симметрическое расширение самосопряженного оператора обязано совпадать с ним, то одновременно доказана формула $H_{0}=P \oplus Q$ (при выполнении условий теоремы 1 ). В более общей ситуации аналогичная формула будет получена в теореме 4.

1.5. Перейдем к доказательству теоремы 2 . Считая, что функция $b(r)$ удовлетворяет ее условиям, обратимся к записи (28) ДВ $\mathscr{L}_{0}$ с коэффициентом $a(|x|)=$ $|b(|x|)|$ в сферических координатах и рассмотрим соответствующее семейство обыкновенных ДВ $\tau_{l}$ вида $(30), l=0,1,2, \ldots$ При доказательстве теоремы 2 мы опираемся на следуюшие соображения. Предположим, что для некоторого $l$ существует функция

$$
0 \neq y_{l}(r) \in L^{2}\left((0,+\infty), r^{m-1} d r\right)
$$


удовлетворяюшая условию

$$
\left(\left(\tau_{l}[g], y_{l}\right)\right)=-\left(\left(g, y_{l}\right)\right), \quad g(r) \in C_{0}^{\infty}((0,+\infty)),
$$

в котором двойными скобками обозначено скалярное произведение в $L^{2}\left((0,+\infty), r^{m-1} d r\right)$. Так как $a(r)=b(r)$ при $r \in\left(0, r_{0}\right)$, то из равенства $(33)$ с произвольной функцией $g(r) \in C_{0}^{\infty}\left(\left(0, r_{0}\right)\right) \subset C_{0}^{\infty}((0,+\infty))$ следует, чTо

$$
\begin{gathered}
\tilde{\tau}_{l}\left[y_{l}\right](r)=-y_{l}(r), \quad r \in\left(0, r_{0}\right), \\
\tilde{\tau}_{l}=-r^{-(m-1)} \frac{d}{d r} r^{m-1} b(r) \frac{d}{d r}+l(l+m-2) \frac{b(r)}{r^{2}} .
\end{gathered}
$$

Возьмем какой-нибудь гармонический многочлен $P_{l}(x)$ порядка $l$ и свяжем с ним функцию

$$
0 \neq u_{l}(x)=y_{l}(|x|) P_{l}\left(\frac{x}{|x|}\right) \in L^{2}\left(\mathbb{R}^{m}\right) .
$$

Пусть $f(x) \in C_{0}^{\infty}\left(\mathbb{R}^{m}\right)$. Зафиксировав число $\rho \in\left(0, r_{0}\right)$, представим эту функцию в виде

$$
\begin{gathered}
f(x)=f^{(1)}(x)+f^{(2)}(x), \\
f^{(1)}(x) \in C_{0}^{\infty}\left(B_{\rho}\right), \quad f^{(2)}(x) \in C_{0}^{\infty}\left(\mathbb{R}^{m} \backslash\{0\}\right) .
\end{gathered}
$$

Если перейти к сферическим координатам $r, \theta$ в скалярном произведении $\left(\mathscr{L}_{0}[f], u_{l}\right)$ в $L^{2}\left(\mathbb{R}^{m}\right)$, то с учетом формулы (28), представления (36), симметричности оператора $\Delta_{\theta}$ в $L^{2}\left(S_{1}, d \sigma\right)$, а также того, что для выражения $h_{l_{0}}(\theta)$ функции $P_{l}\left(\frac{x}{|x|}\right)$ через $\theta$ справедливо равенство (29), получим формулу

$$
\begin{aligned}
\left(\mathscr{L}_{0}[f], u_{l}\right)= & \int_{G}\left(\left(\tilde{\tau}_{l}\left[g_{\theta}^{(1)}\right], y_{l}\right)\right) h_{l}(\theta) D(\theta) d \theta \\
& +\int_{G}\left(\left(\tilde{\tau}_{l}\left[g_{\theta}^{(2)}\right], y_{l}\right)\right) h_{l}(\theta) D(\theta) d \theta=K^{(1)}+K^{(2)},
\end{aligned}
$$

в которой приняты обозначения: $G$ - для области изменения углового параметра $\theta=\left(\theta_{1}, \theta_{2}, \ldots, \theta_{m-1}\right)$, отвечающей единичной сфере $S_{1}, g_{\theta}^{(j)}=g_{\theta}^{(j)}(r)(j=1,2)-$ для выражения функции $f^{(j)}(x)$ через $r, \theta$, рассматриваемого как функция переменной $r \in[0,+\infty)$, зависяшая от $\theta \in G$ как от параметра и, наконец, $D(\theta)$ - для угловой составляющей якобиана $r^{m-1} D(\theta)$ перехода от сферических координат к прямоугольным в $\mathbb{R}^{m}$. При каждом $\theta \in G$ функция $g_{\theta}^{(2)}(r)$ принадлежит классу $C_{0}^{\infty}((0,+\infty))$, так как $f^{(2)}(x) \in C_{0}^{\infty}\left(\mathbb{R}^{m} \backslash\{0\}\right)$. Этот факт вместе с формулой $(33)$, рассматриваемой с функцией $g(r)=g_{\theta}^{(2)}(r)$, влечет за собой второе из равенств

$$
K^{(1)}=-\left(f^{(1)}, u_{l}\right), \quad K^{(2)}=-\left(f^{(2)}, u_{l}\right) .
$$

Первое из них может быть получено с помощью интегрирования по частям в подынтегральном выражении интеграла $K^{(1)}-$ скалярном произведении $\left(\left(\tilde{\tau}_{l}\left[g_{\theta}^{(1)}\right], y_{l}\right)\right)$ в $L^{2}\left((0,+\infty), r^{m-1} d r\right)$, если выполнено дополнительное условие

$$
\lim _{r \rightarrow 0} r^{m-1} y_{l}(r)=\lim _{r \rightarrow 0} r^{m-1} y_{l}^{\prime}(r)=0 .
$$


Нетрудно проверить, что оно влечет за собой равенство нулю внеинтегральных членов, возникаюших при переброске в указанном скалярном произведении ДВ $\tilde{\tau}_{l}$ с $g_{\theta}^{(1)}(r)$ на $y_{l}(r)$, и, следовательно, цепочку равенств

$$
\left(\left(\tilde{\tau}_{l}\left[g_{\theta}^{(1)}\right], y_{l}\right)\right)=\left(\left(g_{\theta}^{(1)}, \tilde{\tau}_{l}\left[y_{l}\right]\right)\right)=-\left(\left(g_{\theta}^{(1)}, y_{l}\right)\right)
$$

последнее из которых вытекает из свойства (34) функции $y_{l}(r)$.

Итак, если функция (52) удовлетворяет условиям $(33),(38)$, то при любой $f(x) \in$ $C_{0}^{\infty}\left(\mathbb{R}^{m}\right)$ справедливы равенства $K^{(j)}=-\left(f^{(j)}, u_{l}\right), j=1,2$, означающие (в силу формул (36) и (37)), что функция $u_{l}(x)$ вида (35) обладает свойством

$$
\left(\mathscr{L}_{0}[f], u_{l}\right)=-\left(f, u_{l}\right), \quad f \in C_{0}^{\infty}\left(\mathbb{R}^{m}\right)
$$

Сушествование функции $u_{l}(x)$ с таким свойством означает, что значения индексов дефекта неотрицательного оператора $H_{0}$, порожденного ДВ $\mathscr{L}_{0}$, не равны нулю. Чтобы доказать теорему 2 , достаточно установить, что при выполнении ее условий функция (32), обладающая свойствами (33), (38), существует для бесконечного набора значений индекса $l$. Если это утверждение доказано, то соответствующие функции (35) будут образовывать бесконечную линейно независимую совокупность, члены которой удовлетворяют условию (39), и, следовательно, оператор $H_{0}$ будет иметь бесконечные индексы дефекта.

1.6. Пусть $U_{l}$ - симметрический и неотрицательный минимальный оператор в $L^{2}\left((0,+\infty), r^{m-1} d r\right)$, порожденньй ДВ $\tau_{l}(l=0,1,2, \ldots), d_{l}$ - его индекс дефекта в верхней комплексной полуплоскости. Существование для какого-нибудь $l$ функции (32), удовлетворяющей условию (33), равносильно неравенству $d_{l} \geqslant 1$. Более точный результат для бесконечного набора значений индекса $l$ вытекает из следующей леммы.

ЛЕмма 3. Если функиия $b(r)$ удовлетворяет условиям теоремы $2, a(r)=$ $|b(r)|$, то при любом $l \geqslant \max \{2-m / 2,0\}$ справедливо равенство $d_{l}=1$.

Заметим, что условие $l \geqslant \max \{2-m / 2,0\}$ имеет вид $l \geqslant 0$ при $m \geqslant 4$, а при $m=2,3$ оно равносильно условию $l \geqslant 1$.

Чтобы доказать лемму 3 , разделим полуось $(0,+\infty)$ точками $q, s, 0<q$ $<r_{0}<s$, на промежутки $J_{1}=(0, q), J_{2}=(q, s), J_{3}=(s,+\infty)$ и обозначим через $d_{l}^{(k)}, k=1,2,3$, индекс дефекта в верхней комплексной полуплоскости минимального оператора $U_{l}^{(k)}$ в $L^{2}\left(J_{k}, r^{m-1} d r\right)$, порожденного ДВ $\tau_{l}$. Аналог формулы (31) в рассматриваемой ситуации имеет вид

$$
d_{l}=d_{l}^{(1)}+d_{l}^{(2)}+d_{l}^{(3)}-4
$$

Ввиду неотрицательности оператора $U_{l}^{(1)}$ число $d_{l}^{(1)}$ совпадает с размерностью подпространства решений $y(r) \in L^{2}\left(J_{1}, r^{m-1} d r\right)$ уравнения $\left(U_{l}^{(1)}\right)^{*} y=-y$. 
ЗАмечаниЕ 3 . Так как $J_{1} \subset\left(0, r_{0}\right)$, то это подпространство состоит из функций $y(r) \in L^{2}\left(J_{1}, r^{m-1} d r\right)$, удовлетворяющих условию $(34)$ с заменой в нем интервала $\left(0, r_{0}\right)$ на $J_{1}$.

Чтобы найти размерность подпространства в $L^{2}\left(J_{1}, r^{m-1} d r\right)$, указанного в замечании 3 , введем оператор

$$
Q f(r)=r^{(m-1) / 2} f(r)
$$

отображающий изометрически пространство $L^{2}\left(J_{1}, r^{m-1} d r\right)$ на $L^{2}\left(J_{1}\right)=$ $L^{2}\left(J_{1}, d r\right)$. Оператор $V_{l}^{(1)}=Q U_{l}^{(1)} Q^{-1}$ является минимальным оператором в $L^{2}\left(J_{1}\right)$, порожденным ДВ

$$
\sigma_{l}=-\frac{d}{d r} b(r) \frac{d}{d r}+\frac{m-1}{2 r} b^{\prime}(r)+\frac{(m-2+2 l)^{2}-1}{4 r^{2}} b(r)
$$

(мы учли, что $a(r)=b(r)$ на $J_{1}$ в силу свойства $(5)$ функции $\left.a(r)\right)$. Размерности подпространств решений уравнений $\left(U_{l}^{(1)}\right)^{*} y=-y$ и $\left(V_{l}^{(1)}\right)^{*} \eta=-\eta$ совпадают; поэтому для нахождения индекса дефекта $d_{l}^{(1)}$ достаточно найти число линейно независимых решений $\eta(r) \in L^{2}\left(J_{1}\right)$ уравнения

$$
\sigma_{l}[\eta]=-\eta
$$

ЗАмечАниЕ 4 . Из замечания 3 , а также равенства $V_{l}^{(1)}=Q U_{l}^{(1)} Q^{-1}$ следует, что решения $\eta(r) \in L^{2}\left(J_{1}\right)$ уравнения (42) связаны с решениями $y(r) \in$ $L^{2}\left(J_{1}, r^{m-1} d r\right)$ уравнения $\tilde{\tau}_{l}[y]=-y$ (вид ДВ $\tilde{\tau}_{l}$ указан в формуле $\left.(34)\right)$ соотношением

$$
y(r)=r^{-(m-1) / 2} \eta(r), \quad r \in J_{1} .
$$

Замена аргумента $t=-\ln \int_{0}^{r} b^{-1}(\rho) d \rho=t(r)$ и искомой функции $\eta(r)=e^{-t / 2} w(t)$ преобразует уравнение (42) к виду

$$
\begin{gathered}
w^{\prime \prime}-\left[\frac{(m-2+2 l)^{2}}{4}+\varphi(t)\right] w=0, \\
\varphi(t)=\frac{(m-2+2 l)^{2}-1}{4}\left(\frac{b^{2}(r(t))}{r^{2}(t)} e^{-2 t}-1\right) \\
+\left(\frac{m-1}{2 r(t)} b^{\prime}(r(t))+1\right) b(r(t)) e^{-2 t}
\end{gathered}
$$

$(r(t)$ - функция, обратная к $t(r))$; при этом стремлению $r$ к нулю отвечает стремление $t \mathrm{k}+\infty$. Нетрудно проверить, что $\int^{\infty}|\varphi(t)| d t<+\infty$. Хорошо известно (см., например, [9, гл. 11 , следствие 9.2$])$, что при $m-2+2 l \geqslant 1$, т.е. при 
$l \geqslant \max \{(3-m) / 2,0\}$, последнее свойство влечет за собой существование фундаментальной системы решений $w_{l, j}(t), j=1,2$, уравнения (43) с асимптотикой

$$
\begin{array}{ll}
w_{l, 1}(t) \sim e^{\left(\frac{m-2}{2}+l\right) t}, & w_{l, 1}^{\prime}(t) \sim\left(\frac{m-2}{2}+l\right) e^{\left(\frac{m-2}{2}+l\right) t}, \\
w_{l, 2}(t) \sim e^{-\left(\frac{m-2}{2}+l\right) t}, & w_{l, 2}^{\prime}(t) \sim-\left(\frac{m-2}{2}+l\right) e^{-\left(\frac{m-2}{2}+l\right) t}, \quad t \rightarrow+\infty .
\end{array}
$$

Функции $\eta_{l, j}(r)=e^{-t(r) / 2} w_{l, j}(r), j=1,2$, образуют фундаментальную систему решений уравнения (42), асимптотика которых при $r \rightarrow 0$ имеет вид

$$
\begin{array}{ll}
\eta_{l, 1}(r) \sim r^{-\left(\frac{m-3}{2}+l\right)}, & \eta_{l, 1}^{\prime}(r) \sim-\left(\frac{m-3}{2}+l\right) r^{-\left(\frac{m-1}{2}+l\right),} \\
\eta_{l, 2}(r) \sim r^{\frac{m-1}{2}+l}, & \eta_{l, 2}^{\prime}(r) \sim\left(\frac{m-1}{2}+l\right) r^{\frac{m-3}{2}+l} .
\end{array}
$$

Если $l \geqslant \max \{2-m / 2,0\} \geqslant \max \{(3-m) / 2,0\}$, то пространству $L^{2}\left(J_{1}\right)$ принадлежит только $\eta_{l, 2}(r)$. Поэтому $d_{l}^{(1)}=1, l \geqslant \max \{2-m / 2,0\}$.

Перейдем к вычислению константы $d_{l}^{(2)}$. Будем рассматривать оператор $Q$, заданный формулой (41), как действуюший из $L_{2}\left(J_{2}, r^{m-1} d r\right)$ в $L_{2}\left(J_{2}\right)$; очевидно, что он отображает изометрически первое из этих пространств на второе. Индексы дефекта операторов $U_{l}^{(2)}$ и $V_{l}^{(2)}=Q U_{l}^{(2)} Q^{-1}$ совпадают; поэтому для нахождения числа $d_{l}^{(2)}$ достаточно подсчитать индексы дефекта действующего в $L^{2}\left(J_{l}\right)$ оператора $V_{l}^{(2)}$, совпадаюшего с минимальным оператором, отвечающим ДВ

$$
\tilde{\sigma}_{l}=-\frac{d}{d r} a(r) \frac{d}{d r}+\frac{m-1}{2 r} a^{\prime}(r)+\frac{(m-2+2 l)^{2}-1}{4 r^{2}} a(r), \quad a(r)=|b(r)| .
$$

Если функция $b(r)$ удовлетворяет условиям теоремы 2 , то его потенциал

$$
\frac{m-1}{2 r} a^{\prime}(r)+\frac{(m-2+2 l)^{2}-1}{4 r^{2}} a(r)
$$

ограничен на промежутке $J_{2}$ и имеет на нем единственную точку разрыва (первого рода) $r_{0}$. Поэтому индексы дефекта оператора $V_{l}^{(2)}$ равны индексам дефекта минимального оператора $V_{l, 0}^{(2)}$ в $L^{2}\left(J_{2}\right)$, порожденного более простым ДВ $-\frac{d}{d r} a(r) \frac{d}{d r}$, коэффициент которого положителен в точках $r \in J_{2} \backslash\left\{r_{0}\right\}$ и равен нулю в точке $r_{0}$. Так как при условиях теоремы 2 функция $a(r)=|b(r)|$ недифференцируема в точке $r_{0}$, можно говорить только о лево- или правостороннем порядках нуля $r_{0}$ этой функции. Из предполагаемого в теореме 2 условия (9) следует равенство единице каждого из них. В лемме 1 статьи [10] установлено ${ }^{1}$, что последнее свойство влечет за собой равенство $d_{l}^{(2)}=3, l \geqslant 0$.

\footnotetext{
${ }^{1}$ Содержащееся в лемме 1 работы [10] требование непрерывности производной $a^{(4)}(r)$ в точках $r \in J_{2} \backslash\left\{r_{0}\right\}$, в которых $a(r) \neq 0$, связано с методом ее доказательства, основанным на использовании асимптотических формул для решений уравнения $\sigma_{l, 0}[y]=z y, \operatorname{Im} z \neq 0$, при $r \rightarrow r_{0}$. Привлечение дополнительных рассуждений, основанных на методе гиперболического уравнения в спектральной теории, позволяет в указанном требовании заменить $a^{(4)}(r)$ на $a^{(2)}(r)$. Непрерывность $a^{(2)}(r)$ в точках $r \in J_{2} \backslash\left\{r_{0}\right\}$, в которых $a(r) \neq 0$, вытекает из условия (4).
} 
При выполнении условий теоремы 2, из которых следует, что $a(r)=$ $|b(r)|>0$ на $J_{3}=(s,+\infty), a(r) \in C^{2}\left(J_{3}\right)$, значения индексов дефекта минимального оператора $U_{l}^{(3)}$ в $L^{2}\left(J_{3}, r^{m-1} d r\right)$, порожденного ДВ (30) при любом $l \geqslant 0$, равны $(1,1)$ согласно упоминавшемуся ранее результату работы [8]. Поэтому $d_{l}^{(3)}=1$, $l \geqslant 0$.

Чтобы завершить доказательство леммы 3, остается подставить значения $d_{l}^{(1)}=1, d_{l}^{(2)}=3, d_{l}^{(3)}=1, l \geqslant \max \{2-m / 2,0\}$, в формулу (40).

Из леммы 3 следует, что при каждом $l \geqslant \max \{2-m / 2,0\}$ существует единственная (с точностью до отличного от нуля числового коэффициента) функция (32), удовлетворяюшая условию (33), из которого вытекает свойство (34) этой функции. В силу замечания 4 при любом $l \geqslant \max \{2-m / 2,0\}$ на промежутке $J_{1}$ функция $\eta_{l}(r)=r^{(m-1) / 2} y_{l}(r)$ является решением уравнения $(42)$, принадлежашим $L^{2}\left(J_{1}\right)$, т.е. $\eta_{l}(r)$ может отличаться от решения $\eta_{l, 2}(r)$ этого уравнения с асимптотикой (44) только числовым множителем $c \neq 0$. Из формулы (44) следует, что

$$
y_{l}(r)=c r^{l}(1+o(1)), \quad y_{l}^{\prime}(r)=c l r^{l-1}(1+o(1))
$$

при $r \rightarrow 0$; поэтому функция $y_{l}(r)$ удовлетворяет условию (38) наряду с (33). Мы объяснили в п. 1.5, что если при каждом $l \geqslant \max \{2-m / 2,0\}$ сопоставить функции $y_{l}(r)$, обладающей всеми перечисленными свойствами, выражение $u_{l}(x)$ вида (35), то мы получим бесконечный набор линейно независимых функций $u_{l}(x)$, $l \geqslant \max \{2-m / 2,0\}$, удовлетворяющих условию (39) (их линейная независимость вытекает, например, из приведенной выше асимптотики функций $y_{l}(r)$ при $\left.r \rightarrow 0\right)$. Существование такого набора означает справедливость утверждения теоремы 2.

1.7. Дополним теоремы 1 и 2 несколькими замечаниями к ним. Можно получить следующее обобщение результатов, полученных в этих теоремах.

ТЕОрема 3. Пусть множество нулей функиии $b(r) \in C^{2}([0,+\infty))$, удовлетворяющей условию $b(0)>0$, конечно или образует возрастающую последовательность

$$
0<r_{1}<r_{2}<\cdots<r_{n}<\cdots, \quad \lim _{n \rightarrow \infty} r_{n}=+\infty
$$

Тогда индексы дефекта минимального оператора $H_{0}$ в $L^{2}\left(\mathbb{R}^{m}\right)$, порожсденного ДВ (2) с коэффичиентом $a(|x|)=|b(|x|)|$, равны нулю, если порядок каждого нуля функиии $b(r)$ больше или равен двум, и бесконечнь, если по крайней мере один из них имеет порядок, равный единиче.

Теорема 3 доказывается с помошью таких же технических средств, какие были использованы в доказательствах теорем 1,2 . При этом главную роль в обосновании ее утверждения о бесконечности индексов дефекта оператора $H_{0}$ играет, как и в случае теоремы 2 , лемма 1 работы [10].

Условие $b(0)>0$ присутствует в каждой из теорем 1-3. Интересно выяснить, насколько оно необходимо для справедливости полученных в них результатов. Можно проверить, что если $b(r) \in C^{2}([0,+\infty)), b(r)>0$ при $r \in(0,+\infty)$ 
и $b(0)=0$, то минимальный оператор $H_{0}$ в $L^{2}\left(\mathbb{R}^{m}\right)$, порожденньй ДВ $\mathscr{L}_{0}$ (в рассматриваемом случае его коэффициент $a(|x|)$ совпадает с $b(|x|))$, самосопряжен независимо от того, выполняется равенство $b^{\prime}(0)=0$ или не вьполняется. После того как получен этот результат, нетрудно проверить, что теорема 3 остается верна и тогда, когда дополнительно к ее условиям вьполнено равенство $b(0)=0$.

Тот факт, что условия теорем 1,2 могут выполняться в случае недифференцируемости коэффициента ДВ $\mathscr{L}_{0}$ в точке $x=0$ (этот случай имеет место, если $\left.b^{\prime}(0) \neq 0\right)$, говорит о том, что порядок гладкости коэффициента ДВ $\mathscr{L}_{0}$ вне множества его точек вырождения, по-видимому, не влияет на значения индексов дефекта соответствующего минимального оператора $H_{0}$. В то же время в связи с теоремами 1-3 можно предположить, что явление бесконечности индексов дефекта симметрического минимального оператора в $L^{2}\left(\mathbb{R}^{m}\right)$, порожденного вырождаюшимся эллиптическим ДВ $(1)$ с потенциалом $q(x) \equiv 0$, возникает при наличии в $\mathbb{R}^{m}$ по крайней мере одной достаточно гладкой гиперповерхности $\Gamma$, в точках которой матрица $A(x)$ его старших коэффициентов не обладает достаточной гладкостью в направлении нормали к Г (очевидно, что если такое явление обнаружено в случае потенциала $q(x) \equiv 0$, то оно сохраняется и тогда, когда, например, $\left.q(x) \in L^{\infty}\left(\mathbb{R}^{m}\right)\right)$. В подтверждение такой гипотезы можно привести ряд примеров, отличных от рассмотренных в теоремах 2,3 , простейшими из которых являются двумерные ДВ:

$$
-\frac{\partial}{\partial x_{1}}\left|x_{1}\right| \frac{\partial}{\partial x_{1}}-\frac{\partial^{2}}{\partial x_{2}^{2}}, \quad-\frac{\partial}{\partial x_{1}}\left|x_{1}\right| \frac{\partial}{\partial x_{1}}-\frac{\partial}{\partial x_{2}}\left|x_{1}\right| \frac{\partial}{\partial x_{2}}
$$

вырождаюшиеся на прямой $x_{1}=0$. Бесконечность индексов дефекта соответствующих им минимальных операторов в $L^{2}\left(\mathbb{R}^{m}\right)$ можно установить с помощью преобразования Фурье и некоторых фактов, относящихся к специальным функциям.

\section{§2. Признак самосопряженности минимального вырождаюшегося эллиптического оператора с потенциалом, произвольным на бесконечности}

2.1. Результаты, полученные в предыдущем параграфе, говорят о естественности требования принадлежности классу $C^{2}\left(\mathbb{R}^{m}\right)$ старших коэффициентов $a_{j k}(x)$ формально самосопряженного вырождаюшегося эллиптического ДВ $\mathscr{L}$ вида (1) при изучении вопроса об условиях самосопряженности соответствующего минимального оператора $H$ в $L^{2}\left(\mathbb{R}^{m}\right)$. Значительный интерес представляет вопрос о том, насколько зависит свойство самосопряженности оператора $H$ от поведения коэффициентов $a_{j k}(x) \in C^{2}\left(\mathbb{R}^{m}\right)$ и потенциала $q(x)$ ДВ $\mathscr{L}$ при $|x| \rightarrow \infty$. Так как в случае эллиптичности указанного ДВ известные признаки самосопряженности $H$ обязательно содержат ограничения на поведение $a_{j k}(x)$ и $q(x)$ в окрестности бесконечно удаленной точки (см., например, работы [4], [11], [12], [13] и приведенные в них ссылки на литературу), естественно предположить, что и в случае вырождающейся эллиптичности ДВ $\mathscr{L}$ условия самосопряженности $H$ должны обладать аналогичным свойством. Результаты, относящиеся к этому случаю, в которых 
указываются ограничения на $a_{j k}(x)$ и $q(x)$ при $|x| \rightarrow \infty$, достаточные для самосопряженности оператора $H$, получены в работах [14], [15, гл. 2, § 15], [16].

В настояшем параграфе приводится признак самосопряженности минимального оператора в $L^{2}\left(\mathbb{R}^{m}\right)$, порожденного вырождающимся эллиптическим ДВ $\mathscr{L}$ (теорема 4), отличаюшийся от подобных результатов тем, что в нем указываются ограничения на матрищу старших коэффициентов указанного ДВ, выполнение которых обеспечивает самосопряженность оператора $H$ при произвольном поведении потенциала $q(x) \in L_{\mathrm{loc}}^{\infty}\left(\mathbb{R}^{m}\right)$ в окрестности бесконечности. Теорему 4 можно рассматривать как вырожденный вариант хорошо известных признаков Хартмана-Исмагилова самосопряженности минимального эллиптического дифференциального оператора в $L^{2}\left(\mathbb{R}^{m}\right)$, условия которых задаются на неограниченно расширяющейся последовательности непересекающихся телесных слоев.

Зафиксируем числовую последовательность $\left\{r_{l}\right\}_{l=0}^{\infty}$, обладающую свойствами

$$
r_{0}=0, \quad r_{l}<r_{l+1}, \quad \lim _{l \rightarrow \infty} r_{l}=+\infty
$$

Свяжем с ней последовательность областей

$$
U_{1}=B_{r_{1}}, \quad U_{l}=B_{r_{l}} \backslash B_{r_{l-1}}, \quad l \geqslant 2 .
$$

Заметим, что при $l \geqslant 2$ области $U_{l}$ являются шаровыми слоями $r_{l-1}<|x|<r_{l}$. Если задать ДВ $\mathscr{L}$ вида (1), то наряду с минимальным оператором $H$ в $L^{2}\left(\mathbb{R}^{m}\right)$ в каждом из пространств $L^{2}\left(U_{l}\right), l \geqslant 1$, оно порождает минимальный оператор, который в дальнейшем обозначается символом $H_{l}$. Напомним, что мы рассматриваем пространства $L^{2}\left(U_{l}\right)$ как подпространства в $L^{2}\left(\mathbb{R}^{m}\right)$; следовательно, $H_{l}$ - это оператор, действуюший в подпространстве $L^{2}\left(U_{l}\right)$ пространства $L^{2}\left(\mathbb{R}^{m}\right)$. Очевидно, что указанные подпространства взаимно ортогональны и справедливо ортогональное разложение $L^{2}\left(\mathbb{R}^{m}\right)=\bigoplus_{l=1}^{\infty} L^{2}\left(U_{l}\right)$.

ТЕОРЕмА 4. Пусть действительная симметрическая матрица $A(x)=$ $\left\{a_{j k}(x)\right\}_{j, k=1}^{m} c$ әлементами

$$
a_{j k}(x) \in C^{2}\left(\mathbb{R}^{m}\right)
$$

обладает свойством неотрицательной определенности:

$$
\sum_{j, k=1}^{\infty} a_{j k}(x) \xi_{j} \xi_{k} \geqslant 0, \quad x \in \mathbb{R}^{m}, \quad \xi=\left(\xi_{1}, \xi_{2}, \ldots, \xi_{m}\right) \in \mathbb{C}^{m}
$$

а действительная функция $q(x)$ удовлетворяет единственному условию

$$
q(x) \in L_{\mathrm{loc}}^{\infty}\left(\mathbb{R}^{m}\right)
$$

Если дополнительно

$$
A(x)=0, \quad x \in S_{r_{l}}, \quad l=1,2, \ldots,
$$


то каждый из операторов $H, H_{l}, l \geqslant 1$, порожденных ДВ $\mathscr{L}$ с указанными матрицей старших коэффициентов $A(x)$ и потенииалом $q(x)$, самосопряжен. При әтом справедливо разлоэсение оператора $H$ в операторную ортогональную сумму:

$$
H=\bigoplus_{l=1}^{\infty} H_{l} .
$$

Пусть ДВ $\mathscr{L}$ удовлетворяет условиям теоремы 4 . Ее доказательство начнем с обсуждения свойств действующего в $L^{2}\left(\mathbb{R}^{m}\right)$ оператора $T=\bigoplus_{l=1}^{\infty} H_{l}$. Его область определения $\mathscr{D}(T)$ состоит из элементов

$$
f=\sum_{l=1}^{\infty} f_{l}, \quad f_{l} \in \mathscr{D}\left(H_{l}\right) \subset L^{2}\left(U_{l}\right), \quad l \geqslant 1,
$$

удовлетворяюших условиям

$$
\sum_{l=1}^{\infty}\left\|f_{l}\right\|^{2}<\infty, \quad \sum_{l=1}^{\infty}\left\|H_{l} f_{l}\right\|^{2}<\infty
$$

а действие оператора $T$ на элементы $f=\sum_{l=1}^{\infty} f_{l} \in \mathscr{D}(T)$ определяется формулой

$$
T f=\sum_{l=1}^{\infty} H_{l} f_{l}
$$

Так как $\mathscr{D}\left(H_{l}\right) \supset C_{0}^{\infty}\left(U_{l}\right), l \geqslant 1$, то

$$
\mathscr{D}(T) \supset \bigoplus_{l=1}^{\infty} C_{0}^{\infty}\left(U_{l}\right)=\mathscr{K}
$$

очевидна плотность множества $\mathscr{K}$ в $L^{2}\left(\mathbb{R}^{m}\right)$.

Предположим, что утверждение теоремы 4 о самосопряженности при каждом $l \geqslant 1$ оператора $H_{l}$, действующего в $L^{2}\left(U_{l}\right)$, доказано. Тогда оператор $T$ самосопряжен в $L^{2}\left(\mathbb{R}^{m}\right)$. Его сужение $T_{0}$ на $\mathscr{K}_{0}$ - совокупность финитных функций класса $\mathscr{K}$ - является также сужением на $\mathscr{K}_{0}$ оператора $H$, т.е. $T_{0} \subset H$. Из определения оператора $T$ и того, что при каждом $l \geqslant 1$ оператор $H_{l}$ является замыканием своего сужения на $C_{0}^{\infty}\left(U_{l}\right)$, следует соотношение $\bar{T}_{0}=T \subseteq H$. Так как оператор $H$ является симметрическим расширением самосопряженного оператора $T$, то оба оператора совпадают (это означает справедливость формулы (51)) и, следовательно, оператор $H$ самосопряжен. Приведенные рассуждения говорят о том, что для доказательства теоремы 4 достаточно установить, что при выполнении ее условий самосопряжен каждый из операторов $H_{l}$, действующих в подпространствах $L^{2}\left(U_{l}\right), l \geqslant 1$, пространства $L^{2}\left(\mathbb{R}^{m}\right)$.

Излагаемое ниже в пाп. 2.2-2.4 доказательство самосопряженности операторов $H_{l}$ основано на следующих соображениях. Предположим, что при некотором $l \geqslant 1$ для любой функции

$$
f(x) \in C_{0}^{\infty}\left(U_{l}\right)
$$


сушествует вектор-функция $v(t)$ параметра $t \in[0,+\infty)$ со значениями в $L^{2}\left(U_{l}\right)$, обладающая свойствами

$$
\begin{gathered}
v(t) \in \mathscr{D}\left(H_{l}\right), \quad t \in[0,+\infty), \\
v(0)=f, \quad v^{\prime}(0)=0, \\
v^{\prime \prime}(t)+H_{l} v(t)=0, \quad t \in[0,+\infty)
\end{gathered}
$$

(они аналогичны свойствам (20)-(22) вектор-функции $v(t)$, использовавшейся в доказательстве утверждения леммы 1 о самосопряженности оператора $Q$ ). Обозначая через $F$ фридрихсово самосопряженное расширение оператора $H_{l}$ (оно сушествует благодаря полуограниченности $H_{l}$, вытекающей из условия (49)), можно переписать свойство (55) в виде

$$
v^{\prime \prime}(t)+F v(t)=0, \quad t \in[0,+\infty) .
$$

Поэтому для $v(t)$ справедливо представление

$$
v(t)=\cos \left(t F^{1 / 2}\right) f, \quad t \in[0,+\infty),
$$

влекушее за собой оценку нормы

$$
\|v(t)\| \leqslant e^{c t}\|f\|, \quad t \in[0,+\infty)
$$

в которой $c=\left|\lambda_{0}\right|^{1 / 2}, \lambda_{0}=\inf F$. По теореме 1 работы [2] из сушествования для каждой функции (52) вектор-функции $v(t)$, обладающей свойствами (53)- $(55)$ и удовлетворяющей приведенной выше оценке нормы, следует самосопряженность операторов $H_{l}$ при всех $l \geqslant 1$. Поэтому утверждение теоремы 4 о самосопряженности операторов $H_{l}$ при всех $l \geqslant 1$ будет доказано, если при каждом $l \geqslant 1$ мы установим существование вектор-функции $v(t)$, удовлетворяющей условиям (53)- $(55)$ при дополнительном предположении (52) относительно начальной функции $f(x)$. Подробную проверку сушествования такой вектор-функции для значений индекса $l \geqslant 2$ мы приведем в пा. 2.2-2.4. Незначительное отличие рассуждений указанной проверки, относящихся к $l \geqslant 2$, от тех, которые нужно было бы провести в случае значения индекса $l=1$, связано с тем, что при $l \geqslant 2$ области $U_{l}$ являются шаровыми слоями, в то время как $U_{1}-$ это шар $|x|<r_{1}$.

2.2. На протяжении пा. $2.2,2.3$ и большей части п. 2.4 значение индекса $l \geqslant 2$ считается фиксированным. Доказательство существования для каждой функции (52) вектор-функции $v(t)$, удовлетворяющей условиям (53)-(55), мы проведем в три этапа. В настоящем пункте изучается свойство конечной скорости распространения решений некоторого вспомогательного вырождающегося гиперболического уравнения с финитными старшими коэффициентами и потенциалом. На втором этапе (в п. 2.3) оно применяется для получения важного вспомогательного результата - леммы 5 . Завершающий этап доказательства существования $v(t)$ содержится в п. 2.4. 
Рассмотрим наряду с ДВ $\mathscr{L}$, удовлетворяющим условиям теоремы 4 , вспомогательное действительное формально самосопряженное ДВ

$$
\tilde{\mathscr{L}}=-\operatorname{div} \tilde{A}(x) \operatorname{grad}+\tilde{q}(x)
$$

связанное с $\mathscr{L}$ соотношениями

$$
\tilde{A}(x)=A(x), \quad \tilde{q}(x)=q(x), \quad x \in B_{r_{l}+\varepsilon_{0}} \backslash B_{r_{l-1}-\varepsilon_{0}},
$$

в которых $\varepsilon_{0}>0$ - достаточно малое число (удобно считать, что $\varepsilon_{0}<$ $\left.\min \left\{r_{l-1}-r_{l-2}, r_{l+1}-r_{l}\right\}\right)$; заметим, что шаровые слои $U_{l}=B_{r_{l}} \backslash B_{r_{l-1}}$ и $B_{r_{l}+\varepsilon_{0}} \backslash B_{r_{l-1}-\varepsilon_{0}}$ связаны соотношением $B_{r_{l}+\varepsilon_{0}} \backslash B_{r_{l-1}-\varepsilon_{0}} \supset U_{l}$. Мы будем предполагать, что старшие коэффициенты $\tilde{a}_{j k}(x)$ ДВ $\widetilde{\mathscr{L}}$ (элементы матрицы $\left.\tilde{A}(x)\right)$ удовлетворяют условиям гладкости и вырождающейся эллиптичности, получающимся путем замены $a_{j k}(x)$ на $\tilde{a}_{j k}(x)$ в формулах $(47)$ и $(48)$, и, кроме того, финитны; будем также считать, что $\tilde{q}(x) \in L_{0}^{\infty}\left(\mathbb{R}^{m}\right)$. ДВ $\widetilde{L}$ удовлетворяет условиям признака А. Девинаца [14] самосопряженности минимального вырождающегося эллиптического оператора второго порядка в $L^{2}\left(\mathbb{R}^{m}\right)$ (с точностью до имеющихся в [14] требований гладкости потенциала $\tilde{q}(x)$, которые в случае, когда

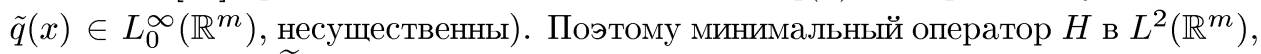
отвечающий ДВ $\widetilde{\mathscr{L}}$, самосопряжен. Из условия $\tilde{q}(x) \in L_{0}^{\infty}\left(\mathbb{R}^{m}\right)$ вытекает полуограниченность этого оператора.

Зафиксируем ограниченную область $G \subset \mathbb{R}^{m}$ и ее открытую окрестность $G_{\rho}$ некоторого радиуса $\rho>0$. Обозначим символом $d_{G}(x)$ расстояние от точки $x \notin G$ до $G$, а символом $\chi(\Omega)$ - действуюший в $L^{2}\left(\mathbb{R}^{m}\right)$ оператор умножения на характеристическую функцию произвольной области $\Omega \subset \mathbb{R}^{m}$. Пусть $\mu(x) \geqslant 0$ - квадратньй корень из наибольшего собственного значения матрицы $\tilde{A}(x)$. Интересующее нас вспомогательное утверждение - лемму 5 - мы получим как следствие такого свойства семейства операторов $\cos \left(t \widetilde{H}^{1 / 2}\right), t \in[0,+\infty)$.

ЛЕмма 4. Предположим, что положительная и непрерывная на $[0, \rho)$ функиия $M(\tau)$ обладает тем свойством, что

$$
\mu(x) \leqslant M(\tau) \quad \text { npu } \quad d_{G}(x)=\tau, \quad \tau \in[0, \rho) .
$$

Тогда для любого $t \in\left[0, t^{*}(\rho)\right)$,

$$
t^{*}(\rho)=\int_{0}^{\rho} M^{-1}(\tau) d \tau
$$

справедлива формула

$$
\cos \left(t \widetilde{H}^{1 / 2}\right) \chi(G)=\chi\left(G_{\rho}\right) \cos \left(t \widetilde{H}^{1 / 2}\right) \chi(G)
$$


ЗАмЕчАнИЕ 5. Из доказательства леммы 4 будет видно, что формула (59) выражает в обобщенной форме свойство конечной скорости распространения решений задачи Коши для вырождаюшегося гиперболического уравнения $u_{t t}+\widetilde{\mathscr{L}}[u]=0$ с начальными данными $u(x, 0)=f(x), u_{t}(x, 0)=0$, в которых $f(x)$ - функция, удовлетворяюшая условию supp $f \subset G$.

ДокАЗАТЕЛЬСТво ЛЕммЫ 4. Рассмотрим последовательности эллиптических ДВ

$$
\tilde{\mathscr{L}}_{n}=\widetilde{\mathscr{L}}+\frac{1}{n} \Delta, \quad n=1,2, \ldots
$$

$\left(\Delta\right.$ - оператор Лапласа в $\left.\mathbb{R}^{m}\right)$; при каждом $n \geqslant 1$ обозначим через $\widetilde{H}_{n}$ минимальньй оператор в $L^{2}\left(\mathbb{R}^{m}\right)$, порожденньй ДВ $\tilde{\mathscr{L}}_{n}$. Вытекающие из определения ДВ $\widetilde{\mathscr{L}}$ принадлежность старших коэффициентов ДВ $\tilde{\mathscr{L}}_{n}$ классу $C^{2}\left(\mathbb{R}^{m}\right)$ и ограниченность их в $\mathbb{R}^{m}$, а также условие $\tilde{q}(x) \in L_{0}^{\infty}\left(\mathbb{R}^{m}\right)$ влекут за собой полуограниченность и самосопряженность каждого из операторов $\widetilde{H}_{n}, n \geqslant 1$ (см., например, [12]). Очевидным является неравенство

$$
\inf H_{n} \geqslant \inf H>-\infty, \quad n \geqslant 1
$$

Рассмотрим задачи Коши:

$$
\begin{gathered}
u^{\prime \prime}+\widetilde{H} u=0, \quad u(0)=f, \quad u^{\prime}(0)=0 \\
u^{\prime \prime}+H_{n} u=0, \quad u(0)=f, \quad u^{\prime}(0)=0, \quad n=1,2, \ldots,
\end{gathered}
$$

для обыкновенных линейных дифференциальных уравнений с операторным коэффициентом относительно вектор-функции $u(t)$ со значениями в $L^{2}\left(\mathbb{R}^{m}\right)$. Если $f(x) \in C_{0}^{\infty}\left(\mathbb{R}^{m}\right) \subset D(\widetilde{H}) \cap D\left(\widetilde{H}_{n}\right)$, то вектор-функция $u(t)=\cos \left(t \widetilde{H}^{1 / 2}\right) f$, $t \in[0,+\infty)$, является решением первой из них, а вектор-функция $u_{n}(t)=$ $\cos \left(t \widetilde{H}_{n}^{1 / 2}\right) f$ - второй. Функции

$$
u(x, t)=u(t)(x), \quad u_{n}(x, t)=u_{n}(t)(x), \quad(x, t) \in \mathbb{R}^{m} \times[0,+\infty),
$$

можно рассматривать как обобшенные решения задачи Коши с начальными данными

$$
u(x, 0)=f(x), \quad u_{t}(x, 0)=0
$$

соответственно для вырождающегося гиперболического уравнения $u_{t t}+\widetilde{\mathscr{L}}[u]=0$ и гиперболического уравнения $u_{t t}+\widetilde{\mathscr{L}}_{n}[u]=0$. Операторы $\cos \left(t \widetilde{H}_{n}^{1 / 2}\right)$ и $\cos \left(t \widetilde{H}^{1 / 2}\right)$ ограничены при каждом $t \in[0,+\infty)$ в силу полуограниченности операторов $\widetilde{H}_{n}, \widetilde{H}$ и связаны предельным соотношением

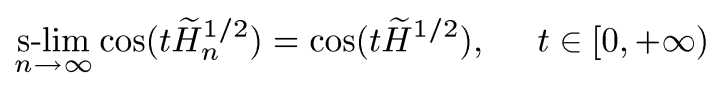

(s-lim - сильный предел последовательности ограниченных операторов в $L^{2}\left(\mathbb{R}^{m}\right)$ ). Чтобы убедиться в его справедливости, воспользуемся очевидным равенством 
$\mathrm{s}^{-} \lim _{n \rightarrow \infty} \widetilde{H}_{n} f=\widetilde{H} f$ для любой функции $f(x) \in C_{0}^{\infty}\left(\mathbb{R}^{m}\right)$. Так как каждый из операторов $\widetilde{H}, \widetilde{H}_{n}, n \geqslant 1$, является замыканием своего сужения на $C_{0}^{\infty}\left(\mathbb{R}^{m}\right)$, то по $[17$, гл. 8 , теорема 8.25$]$ это равенство влечет за собой сильно резольвентную сходимость $\widetilde{H}_{n}$ к $\widetilde{H}$ при $n \rightarrow \infty$. По $[17$, гл. 8, теорема 8.20$]$ из последнего свойства следует сильная сходимость последовательности операторов $\varphi\left(\widetilde{H}_{n}\right)$ к $\varphi(\widetilde{H})$ для любой непрерывной и ограниченной (на оси спектрального параметра $\lambda$ ) скалярной функции $\varphi(\lambda)$. Для получения формулы $(61)$ достаточно взять функцию $\varphi(\lambda)$, совпадающую $c \cos \left(t \lambda^{1 / 2}\right)$ при $\lambda \geqslant \inf \widetilde{H}=\lambda_{0}$ и равную $\cos \left(t \lambda_{0}^{1 / 2}\right)$ при $\lambda<\lambda_{0}$. Свойство конечной скорости распространения решения $u_{n}(x, t)$ гиперболической задачи Коши

$$
u_{t t}+\tilde{\mathscr{L}}[u]=0, \quad u(x, 0)=f(x), \quad u_{t}(x, 0)=0
$$

позволяет найти для ограниченной области $G$ и ее окрестности $G_{\rho}$ такое число

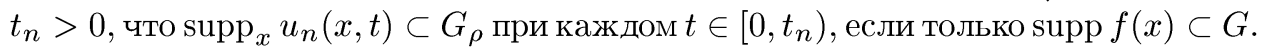
Это свойство равносильно операторному равенству

$$
\cos \left(t \widetilde{H}_{n}^{1 / 2}\right) \chi(G)=\chi\left(G_{\rho}\right) \cos \left(t \widetilde{H}_{n}^{1 / 2}\right) \chi(G), \quad t \in\left[0, t_{n}\right) .
$$

Используя результаты, содержащиеся в $[4$, гл. $1, \S 2$, гл. $2, \S 2]$, можно получить формулу для числа $t_{n}$, имеющую вид

$$
t_{n}=t_{n}(\rho)=\int_{0}^{\rho} M_{n}^{-1}(\tau) d \tau .
$$

В этой формуле $M_{n}(\tau)=\left(M^{2}(\tau)+\frac{1}{n}\right)^{1 / 2}-$ функция, связанная с положительным квадратным корнем $\mu_{n}(x)$ из наибольшего собственного значения матрицы $\tilde{A}(x)+\frac{1}{n} E$ старших коэффициентов ДВ $\tilde{\mathscr{L}}_{n}$ неравенством $0<\mu_{n}(x) \leqslant M_{n}(\tau)$ при $d_{G}(x)=\tau, \tau \in(0, \rho)$.

Так как $\lim _{n \rightarrow \infty} M_{n}(\tau)=M(\tau)$ и $0<M_{n}^{-1}(\tau)<M^{-1}(\tau)$ при каждом $\tau \in$ $[0, \rho)$, то $\lim _{n \rightarrow \infty} t_{n}(\rho)=t^{*}(\rho)$. Поэтому предельный переход при $n \rightarrow \infty$ в равенстве (63) с $t_{n}=t_{n}(\rho)$, основанный на свойстве (61), приводит к справедливости формулы (59) для любого $t \in\left[0, t^{*}(\rho)\right)$.

2.3. Обозначим символом $U_{l}^{(h)}, h \in\left(0, \frac{r_{l}-r_{l-1}}{2}\right)$, шаровой слой $r_{l-1}+h<$ $|x|<r_{l}-h$, являюшийся частью шарового слоя $U_{l}$ (напомним, что мы рассматриваем случай, когда значение индекса $l \geqslant 2$ фиксировано). Доказательство существования для каждой функции (52) вектор-функции $v(t)$, удовлетворяющей условиям (53)-(55), опирается на следующее свойство операторов $\cos \left(t \widetilde{H}^{1 / 2}\right)$, вытекающее из леммы 4.

ЛЕмма 5. Зафиксируем какое-нибудь число $h \in\left(0, \frac{r_{l}-r_{l-1}}{2}\right)$. Следствием условий (47) и (50) теоремы 4 является существование для произвольной константы $N>0$ такого числа $\delta_{N} \in(0, h)$, что при любом $t \in[0, N)$ справедливо равенство

$$
\cos \left(t \widetilde{H}^{1 / 2}\right) \chi\left(U_{l}^{(h)}\right)=\chi\left(U_{l}^{\left(\delta_{N}\right)}\right) \cos \left(t \widetilde{H}^{1 / 2}\right) \chi\left(U_{l}^{(h)}\right) .
$$


ДОКАЗАТЕЛЬСТВО. Проверим то, что при выполнении условий $(47),(50)$ для области $G=U_{l}^{(h)}$ и любого числа $\rho \in(0, h)$ существует функция $M(\tau)$, удовлетворяюшая условиям леммы 4 и такая, что выражение (58) обладает свойством

$$
\lim _{\rho \rightarrow h-0} t^{*}(\rho)=+\infty
$$

Если такая функция найдена, то для каждого $N>0$ можно найти число $\rho_{N} \in(0, h)$, обладающее свойством $t^{*}\left(\rho_{N}\right)>N$. Применяя лемму 4 к указанной области $G$ и числу $\rho=\rho_{N}$, мы получим равенство (59) для произвольного $t \in\left[0, \rho_{N}\right)$ и, в частности, для любого $t \in[0, N)$. Его можно представить в виде (63), если заметить, что окрестностью $G_{\rho_{N}}$ радиуса $\rho_{N}$ области $G=U_{l}^{(h)}$ является шаровой слой $U_{l}^{\left(\delta_{N}\right)}, \delta_{N}=h-\rho_{N}$.

Найдем указанную функцию $M(\tau)$. Согласно формуле (56) неотрицательно определенные матрищы $A(x)$ и $\tilde{A}(x)$ совпадают в шаровом слое $B_{r_{l}+\varepsilon_{0}} \backslash B_{r_{l-1}-\varepsilon_{0}}$, содержащем $U_{l}=B_{r_{l}} \backslash B_{r_{l-1}}$; поэтому $\tilde{A}(x)=0$ на каждой из сфер $S_{r_{n}}, n=l-1, l$, в силу условия (50). Следовательно, любая точка указанных сфер есть точка минимума произвольного диагонального элемента $\tilde{a}_{j j}(x) \geqslant 0$ матрицы $\tilde{A}(x)$, и поэтому дифференциал функции $\tilde{a}_{j j}(x)$ в ней равен нулю. Условие $(47)$ позволяет получить с помошью формулы Тейлора, примененной в каждой точке сфер $S_{r_{n}}$, $n=l-1, l$, оценки

$$
\left|a_{j k}(x)\right| \leqslant c_{1}\left(|x|-r_{n}\right)^{2}
$$

при $r_{n}-\varepsilon<|x|<r_{n}+\varepsilon, n=l-1, l$, с некоторой константой $c_{1}>0$ для достаточно малого числа $\varepsilon>0$ по крайней мере при $k=j$ : справедливость их при $k \neq j$ вытекает из неравенства

$$
\left|a_{j k}(x)\right| \leqslant a_{j j}^{1 / 2}(x) a_{k k}^{1 / 2}(x)
$$

для элементов неотрицательно определенной матрицы $\tilde{A}(x)$. Указанные оценки влекут за собой неравенства

$$
\mu(x) \leqslant c_{2}|| x\left|-r_{n}\right| \quad \text { при } \quad r_{n}-\varepsilon<|x|<r_{n}+\varepsilon, \quad n=l-1, l,
$$

с константой $c_{2}>0$, зависимость которой от $c_{1}$ для нас несущественна. Полагая $G=U_{l}^{(h)}, h \in(0, \varepsilon)$, и учитывая, что множество точек $x$, удовлетворяющих условию $d_{G}(x)=\tau>0$, есть множество $S_{r_{l-1}+h-\tau} \cup S_{r_{l}-h+\tau}$, при любом $\rho \in(0, h)$ получаем как следствие последних неравенств оценку (57) с функцией $M(\tau)=c_{2}(h-\tau)$, для которой выражение (58) имеет вид

$$
t^{*}(\rho)=\frac{1}{c_{2}} \ln \frac{h}{h-\rho}
$$

и поэтому обладает свойством (64). Как объяснено вьше, отсюда следует справедливость леммы 5 при $h<\varepsilon$, где $\varepsilon$ - указанное малое число. Если $h \in\left[\varepsilon, \frac{r_{l}-r_{l-1}}{2}\right)$, то $\chi\left(U_{l}^{(h)}\right)=\chi\left(U_{l}^{\left(\frac{\varepsilon}{2}\right)}\right) \chi\left(U_{l}^{(h)}\right)$, и, следовательно, утверждение леммы 5 для указанных $h$ вытекает из его справедливости для $h=\varepsilon / 2$. 
2.4. Завершим доказательство самосопряженности оператора $H_{l}$, отвечающего значению индекса $l \geqslant 2$. Возьмем произвольную функцию (52) и рассмотрим соответствуюшую вектор-функцию

$$
v(t)=\cos \left(t \widetilde{H}^{1 / 2}\right) f, \quad t \in[0,+\infty) .
$$

Покажем, что она удовлетворяет каждому из условий (53)-(55). Так как $f(x) \in$ $C_{0}^{\infty}\left(U_{l}\right) \subset C_{0}^{\infty}\left(\mathbb{R}^{m}\right) \subset \mathscr{D}(\widetilde{H})$, то $v(t)$ является решением задачи Коши (60); в частности,

$$
v(t) \in \mathscr{D}(\widetilde{H}), \quad t \in[0,+\infty) .
$$

Из условия (52) следует существование числа $h \in\left[0, \frac{r_{l}-r_{l-1}}{2}\right)$ такого, что $\operatorname{supp} f \subset U_{l}^{(h)} ;$ поэтому

$$
f=\chi\left(U_{l}^{(h)}\right) f \quad \text { и } \quad v(t)=\cos \left(t \widetilde{H}^{1 / 2}\right) \chi\left(U_{l}^{(h)}\right) f, \quad t \in[0,+\infty) .
$$

Лемма 5 позволяет утверждать, что для произвольной константы $N>0$ существует число $\delta_{N} \in(0, h)$ такое, что $v(t)=\chi\left(U_{l}^{\left(\delta_{N}\right)}\right) v(t)$ при $t \in[0, N)$. Так как $U_{l}^{\left(\delta_{N}\right)} \subset U_{l}$ для любого $N>0$, то последнее свойство означает, что

$$
\operatorname{supp}_{x} v(t)(x) \subset U_{l}, \quad t \in[0,+\infty) .
$$

В шаровом слое $U_{l}$ ДВ $\mathscr{L}$ и ДВ $\widetilde{\mathscr{L}}$ совпадают в силу условия (56). С учетом этого замечания, а также того, что операторы $H_{l}$ и $\widetilde{H}$ являются замыканиями операторов $C_{0}^{\infty}\left(U_{l}\right) \ni f \rightarrow \mathscr{L}[f]=\widetilde{\mathscr{L}}[f]$ и $C_{0}^{\infty}\left(\mathbb{R}^{m}\right) \ni f \rightarrow \widetilde{\mathscr{L}}[f]$, любая функция

$$
g \in \mathscr{D}(\widetilde{H}), \quad \operatorname{supp} g \subset U_{l},
$$

по-видимому, должна принадлежать $\mathscr{D}\left(H_{l}\right)$ и удовлетворять равенству $\widetilde{H} g=H_{l} g$. Предположим, что такое свойство функции (68) доказано. Тогда из формул (66), (67) вытекает свойство (53) вектор-функции (65), а также равенство $\widetilde{H} v(t)=H_{l} v(t), \quad t \in[0,+\infty)$. Ввиду того, что $v(t)$ является решением задачи Коши (60), это равенство влечет за собой также свойства (54), (55). Таким образом, если справедливо высказанное предположение, то для любой функции (52) вектор-функция (65) обладает свойствами (53)-(55). В конце п. 2.1 объяснено, что наличие таких свойств у рассматриваемой вектор-функции $v(t)$ влечет за собой самосопряженность оператора $H_{l}$. Поэтому утверждение теоремы 4 о самосопряженности оператора $H_{l}$ будет доказано вместе со следующей леммой.

ЛЕмма 6. Если функиия $g(x)$ удовлетворяет условиям (68), то $g \in \mathscr{D}\left(H_{l}\right)$ и справедливо равенство $\widetilde{H} g=H_{l} g$.

ЗАмечАниЕ 6 . Утверждение леммы 6 было бы очевидным, если бы $\mathscr{L}$ и $\widetilde{\mathscr{L}}$ были эллиптическими ДВ. Так как эти ДВ имеют вырождающийся эллиптический характер, оно требует обоснования. 
ДоКАЗАТЕЛЬСТВо ЛЕМмы 6. Условия (68) означают, что сушествует последовательность функций $g_{n}(x) \in C_{0}^{\infty}\left(\mathbb{R}^{m}\right), n \geqslant 1$, обладающая свойствами

$$
\operatorname{s-lim}_{n \rightarrow \infty} g_{n}=g, \quad \underset{n \rightarrow \infty}{\mathrm{s}-\lim } \tilde{\mathscr{L}}\left[g_{n}\right]=\widetilde{H} g
$$

Пусть $\varphi(x) \in C_{0}^{\infty}\left(\mathbb{R}^{m}\right)$. Тогда из формулы

$$
\tilde{\mathscr{L}}\left[\varphi g_{n}\right]=\varphi \tilde{\mathscr{L}}\left[g_{n}\right]+g_{n}(\tilde{\mathscr{L}}[\varphi]-\tilde{q}(x) \varphi)-2\left\langle\tilde{A}(x) \operatorname{grad} g_{n}, \operatorname{grad} \varphi\right\rangle
$$

в которой

$$
\langle\xi, \eta\rangle=\sum_{j=1}^{m} \xi_{j} \eta_{j}, \quad \xi=\left(\xi_{1}, \xi_{2}, \ldots, \xi_{m}\right), \quad \eta=\left(\eta_{1}, \eta_{2}, \ldots, \eta_{m}\right)
$$

видно, что s- $\lim _{n \rightarrow \infty} \widetilde{\mathscr{L}}\left[\varphi g_{n}\right]$ существует, если существует

$$
\underset{n \rightarrow \infty}{\mathrm{S}-\lim }\left\langle\tilde{A}(x) \operatorname{grad} g_{n}, \operatorname{grad} \varphi\right\rangle .
$$

Так как $\left\langle\tilde{A}(x) \operatorname{grad} g_{n}, \operatorname{grad} \varphi\right\rangle=\left\langle\tilde{A}^{1 / 2}(x) \operatorname{grad} g_{n}, \tilde{A}^{1 / 2}(x) \operatorname{grad} \varphi\right\rangle$, причем можно проверить, что элементы матрицы $\tilde{A}^{1 / 2}(x)$ измеримы и ограничены в любой ограниченной области $\Omega \subset \mathbb{R}^{m}$, то для обоснования существования предела (70) достаточно установить сходимость в $L^{2}\left(\mathbb{R}^{m}\right)$ координат вектор-функции $\tilde{A}^{1 / 2}(x) \operatorname{grad} g_{n}$ точки $x \in \mathbb{R}^{m}$ со значениями в $\mathbb{C}^{m}$.

Введем обозначение $|\xi|^{2}=\langle\xi, \xi\rangle$ и рассмотрим очевидное равенство

$$
\begin{aligned}
D_{j k} & =\int_{\mathbb{R}^{m}}\left|\tilde{A}^{1 / 2}(x) \operatorname{grad} g_{j}-\tilde{A}^{1 / 2}(x) \operatorname{grad} g_{k}\right|^{2} d x \\
& =\int_{\mathbb{R}^{m}}\left\langle\tilde{A}(x) \operatorname{grad}\left(g_{j}-g_{k}\right), \operatorname{grad}\left(g_{j}-g_{k}\right)\right\rangle d x \\
& =\left(\tilde{\mathscr{L}}\left[g_{j}\right]-\tilde{\mathscr{L}}\left[g_{k}\right]-\tilde{q}(x)\left(g_{j}-g_{k}\right), g_{j}-g_{k}\right) .
\end{aligned}
$$

С учетом ограниченности и финитности потенциала $\tilde{q}(x)$ в $\mathbb{R}^{m}$ и вытекающей из соотношений (69) ограниченности норм функции $g_{n}, \widetilde{\mathscr{L}}\left[g_{n}\right], n \geqslant 1$, из него следует оценка

$$
D_{j k} \leqslant C\left\|g_{j}-g_{k}\right\|, \quad C-\text { const }>0, \quad 1 \leqslant j, k<\infty,
$$

означающая (в силу первого равенства формулы (69)) справедливость указанного свойства координат вектор-функции $\tilde{A}^{1 / 2}(x) \operatorname{grad} g_{n}$ и вместе с ним существование предела (70). Как объяснено выше, последний факт означает, что s-lim $\lim _{n \rightarrow \infty} \widetilde{\mathscr{L}}\left[\varphi g_{n}\right]$ сушествует; так как $\varphi(x) g_{n}(x) \in C_{0}^{\infty}\left(\mathbb{R}^{m}\right)$, то очевидно, что этот предел равен $\widetilde{H} \varphi g$. Чтобы получить утверждение леммы 6 , следует взять функцию $\varphi(x) \in$ $C_{0}^{\infty}\left(U_{l}\right) \subset C_{0}^{\infty}\left(\mathbb{R}^{m}\right)$, равную единице на носителе функции $g(x)$, удовлетворяющей 
условиям (68). Тогда $\varphi(x) g_{n}(x) \in C_{0}^{\infty}\left(U_{l}\right), \widetilde{\mathscr{L}}\left[\varphi g_{n}\right]=\mathscr{L}\left[\varphi g_{n}\right], n \geqslant 1$ (следствие формулы (56)), и сушествуют

$$
\mathrm{s}_{n \rightarrow \infty} \lim _{n \rightarrow \infty} \varphi g_{n}=\varphi g=g, \quad \operatorname{s}_{n \rightarrow \infty} \lim \mathscr{L}\left[\varphi g_{n}\right]=\mathrm{s}_{n \rightarrow \infty} \lim _{n \rightarrow \infty} \tilde{\mathscr{L}}\left[\varphi g_{n}\right] .
$$

Эти свойства означают, что $g \in \mathscr{D}\left(H_{l}\right)$ и $H_{l} g=\operatorname{s} \lim _{n \rightarrow \infty} \widetilde{\mathscr{L}}\left[\varphi g_{n}\right]=\widetilde{H} \varphi g=\widetilde{H} g$. Лемма 6 доказана. Ее доказательство завершает обоснование самосопряженности операторов $H_{l}$ при $l \geqslant 2$.

Для полного доказательства теоремы 4 осталось установить самосопряженность минимального оператора $H_{1}$ в $L^{2}\left(U_{1}\right)$, порожденного ДВ $\mathscr{L}$, удовлетворяющим ее условиям. Так как граница области $U_{1}$ - шара $B_{r_{1}}$ (сфера $S_{r_{1}}$ ) - имеет более простой характер по сравнению с границами областей $U_{l}, l \geqslant 2,-$ шаровых слоев $B_{r_{l}} \backslash \bar{B}_{r_{l-1}}$, то доказательство самосопряженности оператора $H_{1}$ представляет собой более простой вариант приведенного выше доказательства самосопряженности операторов $H_{l}$ при $l \geqslant 2$. На перечислении незначительных изменений, которые нужно внести в него, чтобы установить самосопряженность оператора $H_{1}$, мы не останавливаемся.

2.5. В заключение укажем на связь теоремы 4 с интересным явлением неустойчивости свойства несамосопряженности действующего в $L^{2}\left(\mathbb{R}^{m}\right)$ минимального оператора, порожденного ДВ $\mathscr{L}$ вида (1), относительно вариации его старших коэффициентов на множестве сколь угодно малой положительной меры в $\mathbb{R}^{m}$, а также приведем другие замечания к этой теореме.

Зафиксируем какую-нибудь числовую последовательность $\left\{r_{l}\right\}_{l=1}^{\infty}$ вида (45) и свяжем с ней, а также с произвольным числом $\delta>0$, функцию $\varphi_{\delta}(r) \in$ $C^{\infty}([0,+\infty))$, удовлетворяющую условиям:

1) $\varphi_{\delta}(r) \geqslant 0, r \in[0,+\infty)$;

2) множество нулей функции $\varphi_{\delta}(r)$ совпадает с последовательностью $\left\{r_{l}\right\}_{l=1}^{\infty}$;

3 ) дополнение $J_{\delta}$ к множеству точек $r \in[0,+\infty)$, в которых $\varphi_{\delta}(r)=1$, имеет меру Лебега $\mu\left(J_{\delta}\right)<\delta$ (в качестве $J_{\delta}$ удобно взять счетное объединение достаточно малых окрестностей точек $\left.r_{l}, l \geqslant 1\right)$.

Предположим, что минимальный оператор $H_{1}$, отвечающий некоторому вырождаюшемуся эллиптическому ДВ $\mathscr{L}=\mathscr{L}_{1}$ вида $(1)$ с действительной симметрической матрицей старших коэффициентов $A(x)=A_{1}(x)$ и действительным потенциалом $q(x)$, удовлетворяюшими условиям (47)-(49), несамосопряжен. Положим $A_{2}(x)=\varphi_{\delta}(|x|) A_{1}(x)$ и рассмотрим вырождающееся эллиптическое ДВ $\mathscr{L}_{2}$, получаемое из $\mathscr{L}_{1}$ заменой матрицы старших коэффициентов последнего $A_{1}(x)$ на матрицу $A_{2}(x)$. Тогда матрица старших коэффициентов и потенциал ДВ $\mathscr{L}_{2}$ будут удовлетворять всем условиям теоремы 4 , вследствие чего отвечающий ему минимальньй оператор $H_{2}$ будет самосопряжен. Вместе с тем в силу свойства 3 функции $\varphi_{\delta}(r)$ для любого положительного числа $\varepsilon>0$ число $\delta$ можно выбрать настолько мальм, что ДВ $\mathscr{L}_{1}$ и ДВ $\mathscr{L}_{2}$ будут отличаться друг от друга только на множестве $\Omega_{\varepsilon}$ в $\mathbb{R}^{m}$, мера Лебега которого меньше $\varepsilon$ (в качестве $\Omega_{\varepsilon}$ можно взять бесконечную последовательность шаровых слоев $r_{l}-\delta_{l}<|x|<r_{l}+\delta_{l}, l \geqslant 1$, толшина каждого из которых $2 \delta_{l}$ - достаточно малое число). 
Приведенный пример показывает, что симметрический несамосопряженный оператор $H$ можно преобразовать в самосопряженный с помощью изменения значений старших коэффищиентов соответствующего вырождающегося эллиптического ДВ $\mathscr{L}$ вида (1) на множестве сколь угодно малой положительной меры (с сохранением свойства вырождающейся эллиптичности). Представляет интерес то, что такое же явление неустойчивости свойства несамосопряженности минимального оператора можно обнаружить также в классе эллиптических ДВ $\mathscr{L}$ (ранее в эллиптическом случае была известна неустойчивость указанного свойства по отношению к вариации потенциала ДВ $\mathscr{L}$ на множестве сколь угодно малой положительной меры; см. $[13, \S 3])$. Для его обоснования достаточно иметь утверждение, обеспечивающее самосопряженность минимального оператора $H$ в $L^{2}\left(\mathbb{R}^{m}\right)$, порожденного эллиптическим ДВ $\mathscr{L}$ с произвольным на бесконечности потенциалом $q(x)$ при некоторых (определяемых поведением $q(x)$ при $|x| \rightarrow \infty$ ) ограничениях, накладьваемых на его старшие коэффициенты только на расширяюшейся последовательности шаровых слоев $V_{l}=B_{d_{l}} \backslash \bar{B}_{c_{l}}, d_{l}<c_{l}<d_{l+1}(l \geqslant 1), \lim _{l \rightarrow \infty} c_{l}=+\infty$, мера объединения которых сколь угодно мала. Такое утверждение (его формулировка громоздка, и поэтому мы ее не приводим) можно получить по крайней мере в случае достаточной гладкости коэффициентов эллиптического ДВ $\mathscr{L}$ как следствие признака самосопряженности минимального эллиптического дифференциального оператора $H$, вытекающего из теоремы 1 статьи [18].

В связи с теоремой 4 укажем на возможность ее обобшения на ДВ $\mathscr{L}$, не обладающее свойством вырождающейся эллиптичности во всем пространстве $\mathbb{R}^{m}$. Зафиксируем какую-нибудь последовательность натуральных чисел $\left\{n_{l}\right\}_{l=1}^{\infty}$, с помощью которой по заданному вырождающемуся эллиптичному ДВ $\mathscr{L}$, удовлетворяющему всем условиям теоремы 4 , построим новое ДВ

$$
\tilde{\mathscr{L}}=-\operatorname{div} \tilde{A}(x) \operatorname{grad}+q(x)
$$

с матрищей старших коэффициентов $\tilde{A}(x)$, связанной с матрищей старших коэффициентов $A(x)$ ДВ $\mathscr{L}$ соотношениями

$$
\tilde{A}(x)=(-1)^{n_{l}} A(x), \quad x \in \bar{U}_{l}, \quad l \geqslant 1,
$$

определяюшими матрицу $\tilde{A}(x)$ однозначно. Минимальный оператор в $L^{2}\left(\mathbb{R}^{m}\right)$, порожденный ДВ $\widetilde{\mathscr{L}}$, обозначим через $\widetilde{H}$. Так же, как в доказательстве теоремы 4 из самосопряженности операторов $H_{l}$ были выведены самосопряженность оператора $H$ и формула (51), может быть установлена самосопряженность оператора $\widetilde{H}$ вместе с формулой

$$
\widetilde{H}=\bigoplus_{l=1}^{\infty}(-1)^{n_{l}} H_{l}
$$

Такое обобщение теоремы 4 интересно в связи с тем, что в случае, когда последовательность $\left\{n_{l}\right\}_{l=1}^{\infty}$ содержит натуральные числа разной четности, ДВ $\widetilde{\mathscr{L}}$ не обладает свойством вырождаюшейся эллиптичности в $\mathbb{R}^{m}$. 
Теорема 4 остается верна, если ее условие (50) выполняется не на последовательности сфер $S_{r_{l}}, \quad l \geqslant 1$, а на неограниченно расширяюшейся последовательности компактных замкнутых достаточно гладких гиперповерхностей $\Gamma_{l}, l \geqslant 1$, в $\mathbb{R}^{m}$ (при этом шаровые слои $U_{l}, l \geqslant 2$, преобразуются в телесные слои, лежащие между ними). Доказательство этого утверждения отличается от доказательства теоремы 4 деталями технического характера. Заметим, что некоторые из рассуждений, используемых в доказательстве теоремы 4 , позволяют сделать вывод о том, что при условии гладкости (47) старших коэффициентов вырождающегося эллиптического ДВ $\mathscr{L}$ вида (1) наличие в $\mathbb{R}^{m}$ компактной замкнутой достаточно гладкой гиперповерхности $\Gamma$, во всех точках которой матрица старших коэффициентов $A(x)$ равна нулю, позволяет представить соответствующий минимальный оператор $H$ в $L^{2}\left(\mathbb{R}^{m}\right)$ в виде ортогональной операторной суммы $H_{1} \oplus H_{2}$, слагаемые которой отвечают соответственно компактной внутренности $\Omega$ и некомпактной внешности $\mathbb{R}^{m} \backslash \bar{\Omega}$ указанной гиперповерхности. При этом $H_{1}$ - это самосопряженный минимальньй оператор в $L^{2}(\Omega)$, а $H_{2}$ - симметрический (не всегда самосопряженный) минимальньй оператор в $L^{2}\left(\mathbb{R}^{m} \backslash \bar{\Omega}\right)$, порожденные ДВ $\mathscr{L}$.

В заключение сделаем замечание, относяшиеся к интересному результату признаку А. Девинаца [14] самосопряженности минимального оператора $H$, порожденного вырождаюшимся эллиптическим ДВ $\mathscr{L}$ вида (1) (он сыграл важную роль в доказательстве утверждения теоремы 4 о самосопряженности операторов $H_{l}$ ). Как показывает теорема 2 , присутствующее в этом признаке условие принадлежности классу $C^{2}\left(\mathbb{R}^{m}\right)$ старших коэффициентов ДВ $\mathscr{L}$ не может быть сушественно ослаблено. Вместе с тем ограничения, накладываемые в работе [14] на поведение при $|x| \rightarrow \infty$ старших коэффициентов и потенциала ДВ $\mathscr{L}$, являются слишком жесткими. С помошью метода гиперболического уравнения, используемого в доказательствах теорем 1 и 4, их можно значительно ослабить. Можно показать, что метод гиперболического уравнения позволяет обобщить на случай вырождающихся эллиптических ДВ второго порядка со старшими коэффициентами класса $C^{2}\left(\mathbb{R}^{m}\right)$ и потенциалом класса $L_{\text {loc }}^{\infty}\left(\mathbb{R}^{m}\right)$ ряд наиболее сильных признаков самосопряженности минимального оператора (с условиями типа Титчмарша-Сирса, Хартмана-Исмагилова), известных в эллиптическом случае.

\section{Список литературы}

1. Орочко Ю. Б. О свойстве глобальной конечной скорости распространения эллиптического дифференциального выражения второго порядка // Дифференц. уравн. Т. 18. № 10. C. $1764-1772$.

2. Орочко Ю. Б. Метод операторного косинуса в задаче о существенной самосопряженности неполуограниченного симметрического оператора // Укр. матем. журн. 1981. Т. 33. № 3. C. 348-355.

3. Соболев С. Л. Некоторые применения функционального анализа в математической физике. Л.: Изд-во ЛГУ, 1950.

4. Орочко Ю. Б. Метод гиперболического уравнения в теории операторов типа Шрёдингера с локально интегрируемым потенциалом // УМН. 1988. Т. 43. Вып. 2 (260). С. 43-86.

5. Шубин М. А. Псевдодифференциальные операторы и спектральная теория. М.: Наука, 1978. 
6. Рид М., Саймон Б. Методы современной математической физики. Т. 2. Гармонический анализ. Самосопряженность. М.: Мир, 1978.

7. Данфорд Н., Швари, Джс. Т. Линейные операторы. Спектральная теория. М.: Мир, 1966.

8. Рофе-Бекетов Ф. С. Замечание в связи с многомерным обобщением теоремы Г. Вейля о самосопряженности // Теория функций, функц. анализ и их прилож. (Республиканский межведомств. научн. сб., г. Харьков). 1989. Вып. 52. С. 88-90.

9. Хартман $\Phi$. Обыкновенные дифференциальные уравнения. М.: Мир, 1970.

10. Орочко Ю. Б. Примеры симметрических дифференциальных операторов на прямой с бесконечными индексами дефекта // Функц. анализ и его прилож. 1994. Т. 28. № 2 . C. $69-72$.

11. Ikebet T., Kato T. Uniqueness of the selfadjoint extension of singular elliptic operators // Arch. Rational Mech. Anal. 1962. V. 9. № 1. P. 77-92.

12. Stetkär-Hansen $H$. A generalization of a theorem of Wienholtz concerning essential selfadjointness of singular elliptic operators // Math. Scand. 1966. V. 9. № 1. P. 108-112.

13. Knowles $I$. On the essential self-adjointness for singular elliptic differential operators // Math. Ann. 1977. V. 227. № 2. P. 152-172.

14. Devinatz A. Selfadjointness of second order degenerate-elliptic operators // Indiana Univ. Math. J. 1978. V. 27. № 2. P. 255-266.

15. Орочко Ю. Б. Гиперболические принципы локализации в теории эллиптических самосопряженных операторов второго порядка: Дис. ... д-ра физ.-мат. наук. М., 1984. С. 312.

16. Перельмутер М. А., Семенов Ю. А. О существенной самосопряженности эллиптических операторов второго порядка с измеримыми коэффициентами // Укр. матем. журн. 1985. T. 37. № 2. C. 191-198.

17. Рид М., Саймон Б. Методы современной математической физики. Т. 1. Функциональный анализ. М.: Мир, 1977.

18. Орочко Ю. Б. Локальная конечная скорость распространения гиперболического уравнения в задаче о самосопряженности степеней эллиптического дифференциального оператора второго порядка // Изв. АН СССР. Сер. матем. 1983. Т. 47. № 2. С. 298-314.

Поступило в редакцию 